\title{
SURFACE SINGULARITY AND CRACK PROPAGATION
}

\author{
ZDENĚK P. BažAnT† and Luis F. Estenssoroł \\ Department of Civil Engineering, Northwestern University Evanston, IL 60201, U.S.A.
}

(Received 7 March 1978; in revised form 13 September 1978; received for publication 31 October 1978)

\begin{abstract}
The three-dimensional singular stress field near the terminal point 0 of the crack front edge at the surface of an elastic body is investigated, using spherical coordinates $r, \theta, \phi$ and assuming all three displacements to be of the form $r^{\lambda} \rho^{p} F(\theta, \phi)$ where $\rho=$ distance from the singularity line (crack front edge or notch edge) and $p=$ given constant. The variational principle governing the displacement distribution on a unit sphere about point 0 , which has previously been obtained from the differential equations of equilibrium, is now derived more directly from potential energy. The previously developed finite element method on the unit sphere is used to reduce the problem to the form $\mathbf{k}(\lambda) X=0$ where $X=$ column matrix of the nodal values of displacements on the unit sphere and $k(\lambda)=$ square matrix, all coefficients of which are quadratic polynomials in $\lambda$. It is proven that the variational principle as well as matrix $k$ must be nonsymmetric, which means that complex eigenvalues $\lambda$ are possible. The dependence of $\lambda$ upon Poisson's ratio $v$ for Mode I cracks whose front edge is normal to the surface is solved numerically and it closely agrees with the analytical solution of Benthem. Previously unavailable solutions for Modes II and III and for cracks (of all modes) with inclined front edge and inclined crack plane are also obtained. By energy flux argument, it is found that the front edge of a propagating crack must terminate at the surface obliquely, at a certain angle whose dependence upon the inclination of the crack plane is also solved. The angle is the same for Modes II and III, but different for Mode I. For this mode, the surface point trails behind the interior of the propagating crack, while for Modes II and III it moves ahead. Consequently, a combination of Mode I with Modes II and III is impossible at the surface terminal point of a propagating crack whose plane is orthogonal. When the plane is inclined, the three stress intensity factors can combine only in certain fixed ratios. The angle of crack edge is a function of the angle of crack plane. Some results with complex $\lambda$ for two-material interfaces are also given.
\end{abstract}

\section{INTRODUCTION}

Crack propagation in thin sheets is undoubtedly influenced by the surface termination of the crack front edge, where the planar elasticity solution for crack tip singularity does not apply and the singular stress field is of three-dimensional nature. A similar situation arises when crack edge intersects a two-material interface. A solution of this problem is of fundamental interest and has been attempted by many without success. Knowledge of the three-dimensional singularity is needed to determine the curved shape of the crack front edge across a thin sheet or plate, and the energy release rate for the advance of the crack front edge as a whole.

An analytical solution for a Mode I crack whose front edge is normal to the surface has been obtained by Benthem [1]. Other analytical solutions for orthogonal cracks $[2,3]$ have also been presented, but they disagree with the results of Benthem [1] as well as the present paper. A numerical method for this type of problem has been recently presented in Ref. [4]. This method is of general applicability and is based on a variational principle for the angular variation of displacements on a unit sphere about the singular point. In Ref. [4], the variational principle was derived from differential equations and finite elements were applied on the unit sphere to obtain a nonlinear eigenvalue problem for the singularity exponent. Some numerical results which agreed with Benthem's analytical solution were also presented.

In the present paper, the variational principle will be derived more directly from the strain energy, and it will be proven that this principle as well as the resulting eigenvalue problem must be nonsymmetric. Complete numerical results for cracks whose front edge is normal to the surface will be given, not only for Mode I cracks but also for Modes II and III cracks. The main objective will, however, be the solution for cracks whose plane as well as front edge are. inclined. It will be shown that a crack which propagates must in fact be inclined and the angles of inclination will be determined. 
The basic ideas of the present solution method, which consist in the separation of variables (postulated in eqns 4 and 9 in the sequel) coupled with a numerical method for a boundary value problem on a unit sphere around the singular point, were enunciated in Ref.[5], which dealt with analogous potential theory problems. Similar techniques for solving two-dimensional and axisymmetric problems have also been employed in Refs. $[6-8,25]$, and a partly similar approach has been used by Swedlow and Karabin [16].

Significant advances, which led to highly accurate analytical solutions, have recently been made in these potential theory problems by Morrison and Lewis[9], and by Keer and Parihar $[10,11]$. The former authors succeed in obtaining a tractable differential equation by virtue of using special coordinates (conical coordinates) suited for the particular problem. Keer and Parihar's method, utilizing spherical coordinates, appears to have broader application and involves the use of Green's functions to formulate the problem in terms of a singular integral. The crucial step is to differentiate this integral equation to get rid of a constant r.h.s. and obtain an eigenvalue problem, which is then solved numerically by Erdogan and Gupta's method. Keer and Parihar have also extended their very effective, original and elegant solution to some three-dimensional singularities (in the interior of an elastic solid) which are irreducible to potential theory [11]. These solutions provide us with valuable checks.

\section{DERIVATION OF VARIATIONAL PRINCIPLE}

Consider the displacement and stress fields in a small neighborhood of a singular point 0 which is located at the termination of either one smooth singularity line $\overline{\mathbf{0 0 ^ { \prime }}}$ or several such singularity lines (see, e.g. Fig. 1). A spherical coordinate system $(r, \theta, \phi)$ is centered at point 0 , such that the pole direction $(\theta=0)$ coincides with singularity line $00^{\prime}$. Displacements in the $r, \theta$ and $\phi$ directions will be denoted as $u, v$ and $w$, respectively, and will be considered to be small. The material is assumed to be linearly elastic, characterized by Young's modulus $E$ and Poisson's ratio $\nu$. The strain energy within volume $V$ of the body is[12]

$$
\begin{aligned}
U & =\int_{V} \Phi \mathrm{d} r \mathrm{~d} \theta \mathrm{d} \phi \\
\Phi & =\frac{C}{2}\left\{Q\left(u_{r}+\frac{1}{r} v_{\theta}+\frac{2}{r} u+\frac{1}{r \sin \theta} w_{\phi}+\frac{1}{r} v \cot \theta\right)^{2}\right. \\
& +2\left[u_{r}^{2}+\left(\frac{1}{r} v_{\theta}+\frac{1}{r} u\right)^{2}+\left(\frac{1}{r \sin \theta} w_{\phi}+\frac{1}{r} v \cot \theta+\frac{1}{r} u\right)^{2}\right] \\
& +\left(v_{r}-\frac{1}{r} v+\frac{1}{r} u_{\theta}\right)^{2}+\left(\frac{1}{r} w_{\theta}-\frac{1}{r} w \cot \theta+\frac{1}{r \sin \theta} v_{\phi}\right)^{2} \\
& \left.+\left(\frac{1}{r \sin \theta} u_{\phi}+w_{r}-\frac{1}{r} w\right)^{2}\right] r^{2} \sin \theta
\end{aligned}
$$

where $Q=\nu /(0.5-\nu), C=E / 2(1+\nu)$, and the subscripts of $u, v, w$ denote partial derivatives; e.g. $v_{\phi}=\partial v / \partial \phi$. We shall now consider displacement variations $\delta u=\epsilon \bar{u}, \delta v=\epsilon \bar{v}, \delta w=\epsilon \bar{w}$ where $\epsilon$ is a variable parameter and $\bar{u}, \bar{v}, \bar{w}$ are any chosen displacement distributions which are sufficiently smooth and satisfy all kinematic boundary conditions. According to the principle of minimum potential energy, the equilibrium state is determined by the variational principle

$$
\begin{aligned}
\delta U=\epsilon\left[\frac{\partial U}{\partial \epsilon}\right]_{\epsilon=0} & =\int_{V}\left(\Phi_{u} \delta u+\Phi_{u_{r}} \delta u_{r}+\cdots+\Phi_{w_{\phi}} \delta w_{\phi}\right) \mathrm{d} r \mathrm{~d} \theta \mathrm{d} \phi \\
& -\int_{S}\left(p_{r} \delta u+p_{\theta} \delta v+p_{\phi} \delta w\right) \mathrm{d} S=0
\end{aligned}
$$

where $p_{n} p_{\theta}, p_{\phi}$ are the distributed loads applied along body surface $S$.

We shall now assume that there are no loads applied at the body surfaces formed by radial rays in a small enough neighborhood of point 0 , and we shall endeavor to determine all possible states, called eigenstates, which satisfy the differential equations of equilibrium and boundary 
conditions on radial rays, but not the boundary conditions that may be prescribed at non-radial surfaces sufficiently remote from point $\mathbf{0}$. According to the principle of superposition, the actual stress state for given boundary conditions can then be expressed as a linear combination of all eigenstates. As far as the eigenstates are concerned, the boundary integral in eqn (3) is irrelevant and may be dropped. Without loss of generality, it may then also be assumed that all body surfaces are formed by radial rays, as must always be true in close vicinity of point 0 .

In analogy to the procedure employed in plane elasticity by Knein[13], Williams [14] and Karp and Karal[15], and similarly to the technique used for three-dimensional potential theory in Ref.[5], it will be assumed that in the vicinity of point 0 the displacement eigenstates can be expressed in the separated form:

$$
u=r^{\lambda} F(\theta, \phi), \quad v=r^{\lambda} G(\theta, \phi), \quad w=r^{\lambda} H(\theta, \phi)
$$

where $\lambda$ is a certain number, in general complex. Now comes a crucial consideration, similar to that made previously for the analogous potential theory problem. If eqn (4) were substituted directly in eqn (3), all terms depending on $r$ would cancel. Then, however, it would be impossible to satisfy the differential equation of equilibrium in the radial direction, because this equation follows by integration by parts with respect to $r$, which would be impossible to implement since all functions of $r$ would have already been eliminated. Therefore, before substituting eqn (4) it is necessary to integrate eqn (3) by parts with respect to only $r$ :

$$
\begin{aligned}
\delta U & =C \int_{V}\left\{\left[\Phi_{u}-\frac{\mathrm{d}}{\mathrm{d} r}\left(\Phi_{u_{r}}\right)\right] \delta u+\phi_{u_{\theta}} \delta u_{\theta}+\phi_{u_{\phi}} \delta u_{\phi}+\left[\phi_{v}-\frac{\mathrm{d}}{\mathrm{d} r}\left(\Phi_{v_{r}}\right)\right] \delta v\right. \\
& \left.+\phi_{v_{\theta}} \delta v_{\theta}+\phi_{v_{\phi}} \delta v_{\phi}+\left[\Phi_{w}-\frac{\mathrm{d}}{\mathrm{d} r}\left(\phi_{w_{r}}\right)\right] \delta w+\Phi_{w_{\theta}} \delta w_{\theta}+\Phi_{w_{\phi}} \delta w_{\phi}\right\} \mathrm{d} r \mathrm{~d} \theta \mathrm{d} \phi=0
\end{aligned}
$$

in which the boundary term has been omitted as justified before. Substituting here eqn (4), and introducing the notations

$$
\begin{aligned}
\Phi_{F}= & C\left\{[Q(1-\lambda)+2]\left[(\lambda+2) F+G_{\theta}+G \cot \theta+\frac{1}{\sin \theta} H_{\phi}\right]-2 \lambda(\lambda+2)\right\} \\
\Phi_{F_{\theta}}= & C\left[(\lambda-1) G+F_{\theta}\right] ; \quad \Phi_{F_{\phi}}=C \frac{1}{\sin \theta}\left[\frac{1}{\sin \theta} F_{\phi}+(\lambda-1) H\right] \\
\Phi_{G}= & C\left\{\left[(Q+2)\left((\lambda+2) F+G_{\theta}+G \cot \theta+\frac{1}{\sin \theta} H_{\phi}\right)-2\left(G_{\theta}+F\right)-2 \lambda F\right] \cot \theta\right. \\
& \left.-2\left(F_{\theta}-G\right)-\lambda(\lambda+1) G-\lambda F_{\theta}\right\} \\
\Phi_{G_{\theta}}= & C\left\{Q\left[(\lambda+2) F+G_{\theta}+G \cot \theta+\frac{1}{\sin \theta} H_{\phi}\right]+2\left(G_{\theta}+F\right)\right\} \\
\Phi_{G_{\phi}}= & \frac{C}{\sin \theta}\left(H_{\theta}-H \cot \theta+\frac{1}{\sin \theta} G_{\phi}\right) \\
\Phi_{H}= & C\left\{\left[\left(H_{\theta}-H \cot \theta+\frac{1}{\sin \theta} G_{\phi}\right) \cot \theta+2\left(\frac{1}{\sin \theta} F_{\phi}-H\right)+\lambda(\lambda+1) H+\frac{\lambda}{\sin \theta} F_{\phi}\right]\right\} \\
\Phi_{H_{\theta}}= & C\left\{H_{\theta}-H \cot \theta+\frac{1}{\sin \theta} G_{\phi}\right\} \\
\Phi_{H_{\phi}}= & \frac{C}{\sin \theta}\left\{Q\left[(\lambda+2) F+G_{\theta}+G \cot \theta+\frac{1}{\sin \theta} H_{\phi}\right]+2\left(\frac{1}{\sin \theta} H_{\phi}+G \cot \theta+F\right)\right\}
\end{aligned}
$$

we obtain

$$
\delta U=\int_{r} r^{2 \lambda} \bar{U} \mathrm{~d} r=0
$$


where

$$
\begin{aligned}
\bar{U} & =\iint_{A}\left(\Phi_{F} \delta F+\Phi_{F_{\theta}} \delta F_{\theta}+\Phi_{F_{\phi}} \delta F_{\phi}+\Phi_{G} \delta G+\Phi_{G_{\theta}} \delta G_{\theta}+\Phi_{G_{\phi}} \delta G_{\phi}+\Phi_{H} \delta H\right. \\
& \left.+\Phi_{H_{\theta}} \delta H_{\theta}+\Phi_{H_{\phi}} \delta H_{\phi}\right) \sin \theta \mathrm{d} \theta \mathrm{d} \phi
\end{aligned}
$$

Symbol $A$ denotes the area of the region of the $(\theta, \phi)$ plane occupied by the elastic solid. Note that $\Phi_{F} \ldots \Phi_{H_{\phi}}$ are not partial derivatives of some function $\Phi$.

Equation (7) represents the basic variational equation of the problem; i.e. functions $F, G$ and $H$ are the solutions of the problem if and only if they satisfy eqn (7) for any kinematically admissible variations $\delta F, \delta G, \delta H$.

\section{TREATMENT OF LINE SINGULARITY}

From the three-dimensional singular point 0 there usually emanates a stress singularity line, such as the front edge of a crack. Let this line coincide with the polar ray, $\theta=0$. The displacements near this line usually behave as $(r \rho)^{p}$ such that $r \rho$ represents the distance from the ray $\theta=0$ when $\theta \rightarrow 0$. Possible choices are $\rho=\theta$ or $\rho=\sin \theta$, of which the latter has been used in numerical calculation. (Note, however, that $\rho=\sin \theta$ cannot be used when the angle $\theta=\pi$ is part of the domain considered and no singularity exists at $\theta=\pi$ ) For the crack edge, the values $p=0,1 / 2,1,3 / 2, \ldots$ are generally present, and for notch edges other values of $p$ would apply.

If $p<1$, functions $F, G$ and $H$ may obviously exhibit gradient singularity at $\theta=0$. Then, if these functions are approximated numerically, accuracy and the rate of convergence are adversely affected by the presence of the singularity. From the theory of finite element method for plane problems it is known, for example, that the rate of convergence in presence of square-root singularity is only $O(V(h))$, while in absence of singularity the convergence is quadratic, $O\left(h^{2}\right)[(17), h$ being the element size. Thus, an improvement in accuracy and the rate of convergence may, be expected if one sets

$$
F(\theta, \phi)=\rho^{p} f(\theta, \phi), \quad G(\theta, \phi)=\rho^{p} g(\theta, \phi), \quad H(\theta, \phi)=\rho^{p} h(\theta, \phi) .
$$

Then, if there is only one exponent $p<1$ present in the solution, functions $f, g$ and $h$ may be expected to be free of gradient singularity, in which case a quadratic convergence should take place if functions $f, g$ and $h$ are approximated numerically.

When several exponents $p<1$ are present, the lowest one must be used. This was shown in Ref. [4] as follows. Consider than an exponent $p^{*}$ which differs from the actual value of $p$ is used, i.e. $u \sim r^{\lambda} \theta^{p^{*}} F^{*}(\theta, \phi)$. The stresses then are $\sigma_{i j} \sim \partial u / \partial \theta \sim r^{\lambda} \theta^{p-1} F(\theta, \phi)$ for the exact solution (if $p=0$ is excluded) and $r^{\lambda} \theta^{p^{*}-1} F^{*}(\theta, \phi)$ for the numerical solution. Equating these two expressions for $\sigma_{i j}$, one obtains $F^{*}(\theta, \phi)=\theta^{p-p^{*}} F(\theta, \phi)$ where function $F$ is bounded. If one chooses $p^{*}>p$, function $F^{*}(\theta, \phi)$ can obviously become unbounded as $\theta \rightarrow 0$, which cannot be adequately represented numerically. Thus $p^{*} \leq p$ is necessary, and the best choice is to make $p^{*}$ equal to the lowest exponent $p$ present.

In the case of a crack front edge which terminates orthogonally at body surface, not only $p=1 / 2$ but also $p=0$ is present in the exact solution [1] (and the authors are obliged to Prof. J. P. Benthem of Delft University of Technology for pointing this out). Therefore, it is necessary to take $p=0$, in which case $f=F, g=G$ and $h=H$. Because $p=1 / 2$ is present, too, the convergence rate must be less than quadratic. For crack edges which terminate at the surface obliquely, the exact solution is not known. Nevertheless, exponent $p=0$ was assumed for all numerical calculations of cracks that terminate at the surface. However, for the case of a sharp corner that lies within the solid on the front edge of a planar crack, the exact solution (for Mode I) is known and involves only $p=1 / 2$; in that case $p=1 / 2$ was, therefore, considered in numerical calculations, and the practical convergence rate indeed appeared to be quadratic, $O\left(h^{2}\right)$. 


\section{FINITE ELEMENT SOLUTION}

Analogous problems of potential theory have previously been solved by finite difference method in $(\theta, \phi)$ plane. In the elasticity problem, however, formulation of the stress-free boundary conditions in spherical coordinates by finite differences would be rather complicated, and further complexities would arise from the use of a nonuniform grid. Thus, it is advantageous to adopt the finite element approach, in which the stress-free boundary conditions are automatically implied.

Choosing a finite element grid in the $(\theta, \phi)$ plane, we may represent the unknown functions $F, G$ and $H$ within each finite element in the form

$$
\begin{aligned}
F(\theta, \phi)=\Sigma_{i} X_{i} F^{i}, & F^{i}=\rho^{p} f^{i}(\theta, \phi) \\
G(\theta, \phi)=\Sigma_{i} X_{i} G^{i}, & G^{i}=\rho^{p} g^{i}(\theta, \phi) \\
H(\theta, \phi)=\Sigma_{i} X_{i} H^{i}, & H^{i}=\rho^{p} h^{i}(\theta, \phi)
\end{aligned}
$$

in which $X_{i}(i=1,2, \ldots M)$ are the nodal values of $f(\theta, \phi), g(\theta, \phi), h(\theta, \phi)$ and $f^{i}(\theta, \phi)$, $g^{i}(\theta, \phi), h^{i}(\theta, \phi)$ are given distribution functions within the finite elements, normally chosen as polynomials in $\theta$ and $\phi$. Denoting by $\theta_{m}$ and $\phi_{m}$ the coordinates of $m$ th node, the distribution functions must be chosen such that $f^{i}\left(\theta_{m}, \phi_{m}\right), g^{j}\left(\theta_{m}, \phi_{m}\right)$ and $h^{k}\left(\theta_{m}, \phi_{m}\right)$ equal 1 if $i=3 m-2$, $j=3 m-1$ and $k=3 m$, and equal 0 for all other values of $i, j, k$.

Following the standard procedure in finite element method[17] (see Appendix B), the variational equation (7) leads to the following system of $M$ algebraic linear homogeneous equations for nodal unknowns $X_{i}$ :

$$
\sum_{j=1}^{M} k_{i j}(\lambda) X_{i}=0 \quad(i=1,2, \ldots M)
$$

in which the stiffness coefficients are calculated as:

$$
\begin{aligned}
k_{i j}= & \iint_{A}\left\{\Phi_{F}^{i} F^{i}+\Phi_{F_{\theta}}^{i} F_{\theta}^{j}+\Phi_{F_{\phi}}^{i} F_{\phi}^{j}+\Phi_{G}^{i} G^{j}+\Phi_{G_{\theta}}^{i} G_{\theta}^{j}+\Phi_{G_{\phi}}^{i} G_{\phi}^{i}+\Phi_{H}^{i} H^{i}\right. \\
& \left.+\Phi_{H_{\theta}}^{i} H_{\theta}^{j}+\Phi_{H_{\phi}}^{i} H_{\phi}^{j}\right\} \sin \theta \mathrm{d} \theta \mathrm{d} \phi .
\end{aligned}
$$

Here $F_{\theta}^{j}=\partial F^{i} / \partial \theta$, etc. and $\Phi_{F}^{i}, \ldots \Phi_{H_{\phi}}^{i}$ are given by eqn (6) after the substitutions $F=$ $\rho^{p} f^{i}(\theta, \phi), G=\rho^{p} g^{i}(\theta, \phi)$ and $H=\rho^{p} h^{i}(\theta, \phi)$ are made, which must include the derivatives; e.g. $H_{\theta}$ is replaced by $\partial\left[\rho^{p} h^{i}(\theta, \phi)\right] / \partial \theta$.

Equation (11) is a system of $M$ algebraic linear homogeneous equations for nodal unknowns $X_{i}$. The problem is to find the $\lambda$-values greater than $-1 / 2$, for which eqn (11) has a non-zero solution (eigenvalues). Of main interest is the smallest such $\lambda$-value, which gives the dominant field near point 0 . All stiffness coefficients, not just the diagonal ones, depend on the singularity exponent $\lambda$, and so the eigenvalue problem is a generalized one. It is also nonlinear because $k_{i j}$ depend on $\lambda$ nonlinearly. Various methods of numerical eigenvalue search were discussed in Ref.[5], and method $B$ from p. 234 of Ref. [5] has been adopted for the present problem. It consists in choosing $\lambda$, forcing one specified $X_{k}$-component to equal 1 , and using then a standard equation solving subroutine for banded nonsymmetric equation systems to solve all other $X_{i}$ and the r.h.s. $R_{k}$ of the $k$ th equation. This procedure is then iterated and $\lambda$ is varied according to Newton method until the $\lambda$ value for which $R_{k}=0$ is found. (This method has originally been programmed for solving critical loads of large frameworks [18], which also leads to a problem of the form of eqn 11.) An extended method of solution for problems where $\lambda$ is complex has been described in Ref. [8].

It is interesting to note that stiffness coefficients $k_{i j}(\lambda)$ are quadratic polynomials in $\lambda$. This is because all terms of the variational principle (eqn 8) are quadratic polynomials of $\lambda$ (eqn 6). Accordingly, the matrix of eqn (11) may be written as $\left[k_{i j}\right]=\mathbf{k}=\mathbf{a}+\mathbf{b} \lambda+\mathbf{c} \lambda^{2}$ where $\mathbf{a}, \mathbf{b}, \mathbf{c}$ are real square matrices which are of size $(M \times M)$ and are independent of $\lambda$. So, eqn (11), $\mathbf{k X}=0$, 
takes the form

$$
\mathbf{a X}+\lambda \mathbf{b X}+\lambda^{2} \mathbf{c X}=0
$$

where $\mathbf{X}=$ column matrix of $X_{i}$. This is a quadratic eigenvalue problem.

Equation (13) allows reduction in the number of computations needed when $k_{i j}$ is to be repeatedly evaluated for various $\lambda$-values in an iterative eigenvalue search. It suffices to determine three matrices $\mathbf{a}, \mathbf{b}$ and $\mathbf{c}$, which are independent of $\lambda$, and then evaluate $\mathbf{k}$ for a given $\lambda$ from them.

Equation (13) could further be converted to a linear generalized eigenvalue problem for a matrix of doubled size $(2 M \times 2 M)$; this, however, would not be effective for numerical solution. Furthermore, it is useful to observe that complex eigenvalues of eqn (11) or eqn (13), if any, can occur only in conjugate pairs. This is because $\overline{\mathbf{k}(\lambda) \mathbf{X}}=\overline{\mathbf{k}(\lambda) \mathbf{X}}=\mathbf{k}(\bar{\lambda}) \overline{\mathbf{X}}$ so that if $\mathbf{k}(\lambda) \mathbf{X}=0$ then also $\mathbf{k}(\bar{\lambda}) \overline{\mathbf{X}}=0$, where a superposed bar denotes a complex conjugate.

\section{LACK OF SYMMETRY AND NON-EXISTENCE OF A MINIMUM PRINCIPLE}

As a consequence of the integration by parts with respect to $r$ (eqn 5 ), the integrand of eqn (8) is nonsymmetric, and so is the system of linear equations, eqn (11) $\left(k_{i j} \neq k_{j i}\right)$, as well as the coefficient matrices a, b, $\mathbf{c}$ in eqn (13). This means that it is impossible to find a functional $W$ such that $\delta W=0$ would yield eqn (7), which greatly complicates the numerical solution. For a solid that is elastic this might seem surprising at first. However, a deeper examination reveals that it must be so.

One simple consideration which suggests that eqn (11) may have to be nonsymmetric is as follows: If the matrix were symmetric and eqn (11) represented a standard eigenvalue problem, all roots $\lambda$ would be real. This would be, however, impossible because the same variational principle also holds for plane strain problems with two-material interfaces, in which complex eigenvalues $\lambda$ (oscillating singularities) are known to exist. This contrasts with the analogous potential theory problem, for which a symmetric variational formulation exists [5], and all roots $\lambda$ are indeed real.

To prove that the variational equation must be non-symmetric, it is sufficient to show that it must be so in the special case of plane elasticity, which is obtained by dropping integration over $\theta$ and substituting $\theta=\pi / 2$, and setting $G=v=0$. In that case, the most general quadratic functional involving $F(\phi), H(\phi), F^{\prime}(\phi)=\mathrm{d} F / \mathrm{d} \phi, H^{\prime}(\phi)=\mathrm{d} H / \mathrm{d} \phi$ is

$$
\begin{gathered}
W=\int_{0}^{\phi_{1}}\left[\frac{1}{2}\left(A_{1} F^{2}+A_{2} F^{\prime 2}+A_{3} H^{2}+A_{4} H^{\prime 2}\right)+A_{5} F F^{\prime}+A_{6} F H+A_{7} F H^{\prime}\right. \\
\left.+A_{8} F^{\prime} H+A_{9} F^{\prime} H^{\prime}+A_{10} H H^{\prime}\right] \mathrm{d} \phi
\end{gathered}
$$

The associated Euler equations are

$$
\begin{aligned}
& A_{1} F-A_{2} F^{\prime \prime}+A_{6} H+\left(A_{7}-A_{8}\right) H^{\prime}-A_{9} H^{\prime \prime}=0 \\
& A_{3} H-A_{4} H^{\prime \prime}+A_{6} F-\left(A_{7}-A_{8}\right) F^{\prime}-A_{9} F^{\prime \prime}=0
\end{aligned}
$$

and the corresponding natural boundary conditions at $\phi=0$ or $\phi=\phi_{1}$ are

$$
\begin{aligned}
A_{2} F^{\prime}+A_{5} F+A_{8} H+A_{9} H^{\prime} & =0 \\
A_{4} H^{\prime}+A_{7} F+A_{9} F^{\prime}+A_{10} H & =0 .
\end{aligned}
$$

The actual differential equations for $F$ and $H$, as obtained by substituting $u=r^{\wedge} F$ and $w=r^{\wedge} G$ into the planar differential equations of equilibrium in polar coordinates $r, \phi$ (see Karp and Karal[15]) have the form

$$
\begin{aligned}
& C_{1}\left(a_{0} F+a_{2} F^{\prime \prime}+a_{1} H^{\prime}\right)=0 \\
& C_{2}\left(b_{0} H+b_{2} H^{\prime \prime}+b_{1} F^{\prime}\right)=0
\end{aligned}
$$


and the actual boundary conditions are

$$
\begin{aligned}
& C_{3}\left(c_{1} F^{\prime}-c_{2} H\right)=0 \\
& C_{4}\left(c_{3} H^{\prime}+c_{4} F\right)=0
\end{aligned}
$$

where $C_{1}, C_{2}, C_{3}$ and $C_{4}$ are arbitrary non-zero constants and $a_{0}, a_{1}, a_{2}, b_{0}, b_{1}, b_{2}, c_{1}, \ldots c_{4}$ are certain given constants. Equating the coefficients of all corresponding terms of eqns (15) and (16) with eqns (17) and (18) one obtains a system of 14 linear algebraic equations for $A_{1}, \ldots A_{10}, C_{1}, \ldots C_{4}$. Unknowns $A_{1}, \ldots A_{10}$ can be easily eliminated, which leaves a system of four linear equations for $C_{1}, \ldots C_{4}$ which are homogeneous. The determinant of this equation system is found to equal $\lambda$. Because $\lambda$ cannot be restricted to equal 0 , it follows that $C_{1}, \ldots C_{4}$ cannot be non-zero. Thus, there is no way to make eqns (17), (18) equivalent to eqns (15), (16), which means that a variational functional $W$ does not exist for the plane problem. So, it cannot exist for the three-dimensional problem as well.

The possibility of complex eigenvalues $\lambda$ contrasts with the situation in the analogous problem of the potential theory [5]. That problem can also be written in the form of eqn (13) but it can be simplified to the form $\mathbf{a X}-\kappa \mathbf{d X}=0$ where $\kappa=\lambda(\lambda+1)$, which is a generalized linear eigenvalue problem for $\kappa$. Because a and $\mathbf{d}$ are symmetric positive definite matrices, $\kappa$ is always real and positive. Moreover, because $\lambda=-(1 / 2) \pm((1 / 4)+\kappa)^{1 / 2}$, all eigenvalues $\lambda$ are also real.

\section{ENERGY RESTRICTIONS FOR CRACK PROPAGATING AT SURFACE}

As the crack propagates, energy flows into all points of the crack front edge and is consumed by the process of separation (creation of crack surfaces). The energy fiux near the points of the crack front edge may generally have two components: the flux $E_{0}$ which flows into the crack front edge, and the flux $E_{0}^{\prime}$ which is parallel to the edge.

Energy flux $E_{0}$ may in general be expressed by Rice's $J$-integral [19] over a small circle $L$ of radius $r_{0}$ around the crack front edge:

$$
E_{0}=\oint_{L}\left(\frac{1}{2} \sigma_{i j} \epsilon_{i j} \mathrm{~d} y-n_{j} \sigma_{i j} \frac{\partial u_{i}}{\partial x} r_{0} \mathrm{~d} \phi\right)
$$

in which $\mathrm{d} y=r_{0} \sin \phi \mathrm{d} \phi$ and $\partial / \partial x=\cos \phi\left(\partial / \partial r_{1}\right)-\left(\sin \phi / r_{1}\right)(\partial / \partial \phi), r_{1}=r \rho$. On physical grounds, flux $E_{0}$ must obviously be positive, non-zero and finite (bounded) at all points near the terminal point 0 of crack edge. Furthermore, flux $E_{0}$ may reasonably be expected to be constant along the crack edge [4], assuming that the energy needed for surface creation is the same at all points of the crack front edge; however, this last requirement is not essential for the subsequent deductions.

Near the surface terminal point 0 we have $u_{i} \sim r^{\lambda}$ where $\sim$ denotes proportionality. Hence, $\partial u_{i} / \partial x \sim r^{\lambda} / r \rho \sim r^{\lambda-1}, \epsilon_{i j} \sim \sigma_{i j} \sim r^{\lambda-1}, E_{0} \sim r_{0}^{\lambda-1} r_{0}^{\lambda-1} r_{0}=r_{0}^{2 \lambda-1}$. Thus, for $E_{0}$ to be bounded and non-zero as $r_{0} \rightarrow 0$, it is necessary that $\operatorname{Re}(2 \lambda-1)=0$ or

$$
\operatorname{Re}(\lambda)=1 / 2 \quad \text { (propagating crack). }
$$

for any crack that propagates [4].

Alternatively, we may consider the variation of the stress intensity factor, $K$, along the crack front edge. For $\operatorname{Re}(\lambda)<(1 / 2), K$ approaches $\infty$ as $r \rightarrow 0$ and for $\operatorname{Re}(\lambda)>(1 / 2), \lim K=0$ as $r \rightarrow 0$ (see $c$ in Fig. 9). None of these is physically admissible for a propagating crack, and so we see again that $\operatorname{Re}(\lambda)=(1 / 2)$.

From energy considerations, further restrictions may be deduced. The flux of energy into a sphere of radius $r_{0}$ (centered at the surface terminal point 0 ) as point 0 propagates may be found to be proportional to $r_{0}^{2 \lambda}$. The flux of energy into point 0 is obtained in the limit for $r_{0} \rightarrow 0$, and it must be zero, because the trace of point 0 as it moves is a line, and a line can be associated only with a negligible amount of surface energy. This requires that for a propagating crack $\operatorname{Re}(\lambda)>0$, which is a weaker restriction than the previous one.

For cracks that do not propagate, the only restrictions are that the strain energy within a 
small sphere about point 0 , as well as the strain energy (per unit length of edge) within a small cylinder whose axis coincides with the crack front edge, be integrable. These conditions yield

$$
\operatorname{Re}(p)>0 \text { or } p=0, \text { and } \operatorname{Re}(\lambda)>-\frac{1}{2} \quad \text { (stationary crack). }
$$

\section{DEVELOPMENT OF COMPUTER PROGRAM}

The finite elements were introduced as four-node quadrilaterals in the $(\theta, \phi)$ plane, obtained by mapping of a unit square. The basic distribution (shape) functions $f^{i}(\theta, \phi), g^{i}(\theta, \phi)$ and $h^{i}(\theta, \phi)$ on the original rectangle have been considered as bilinear, i.e. as $a+b \theta+c \phi+\mathrm{d} \theta \phi$. The stiffness coefficients $k_{i j}$ have been evaluated by Gaussian numerical integration, using nine integration points per quadrilateral (see Appendix B).

The program developed (listed in report[20]) is general and capable of handling diverse types of three-dimensional singularities, such as the crack front edge intersecting body surface, for any angle of the crack edge as well as crack plane; the edge of a two-plane or multiple-plane notch (with arbitrarily inclined planes) intersecting a body surface; the apex of a pyramidal notch, consisting of several planes at any angle; the crack front edge intersecting a notch edge or notch apex; the conical notches, possibly with a crack or another notch edge intersecting the cone apex, etc.

To check the program, various simple cases of known solution have been considered [4]. First, various elementary solutions have been substituted into eqn (11) written for a crack whose front edge is normal to body surface, so as to see whether the r.h.s. are indeed approximately zero, as indicated by the condition $\left|\Sigma k_{i j} X_{j}\right| / \Sigma\left|k_{i j} X_{j}\right|<10^{-4}$. These test cases included: (a) three rigid body rotation fields, for which $\lambda=1$ and $p=0$; (b) the displacement for homogeneous uniaxial stress in the direction $\theta=\pi / 2, \phi=0$, for which $\lambda=1, p=0$; (c) the near-edge plane-strain field of Mode I crack for $\nu=0$, for which $\lambda=p=1 / 2$; (d) the same for Mode II crack, for which also $\lambda=p=1 / 2$; (e) homogeneous strain fields with various strain components being non-zero and constant $(\lambda=1, p=0)$; (f) the antiplane (Mode III) near-edge field $(\lambda=p=1 / 2$ ). Cases (e) and (f) do not satisfy all stress boundary conditions, and so in these cases only the fulfillment of the equilibrium equations for the interior nodes was checked.

Furthermore, the program was checked against the known analytical solution for a sharp corner of angle $\alpha$ on the crack front edge of a planar crack within a solid. In this case the stress boundary conditions at the bottom side of the domain in Fig. $1(\theta=\pi / 2)$ must be replaced by
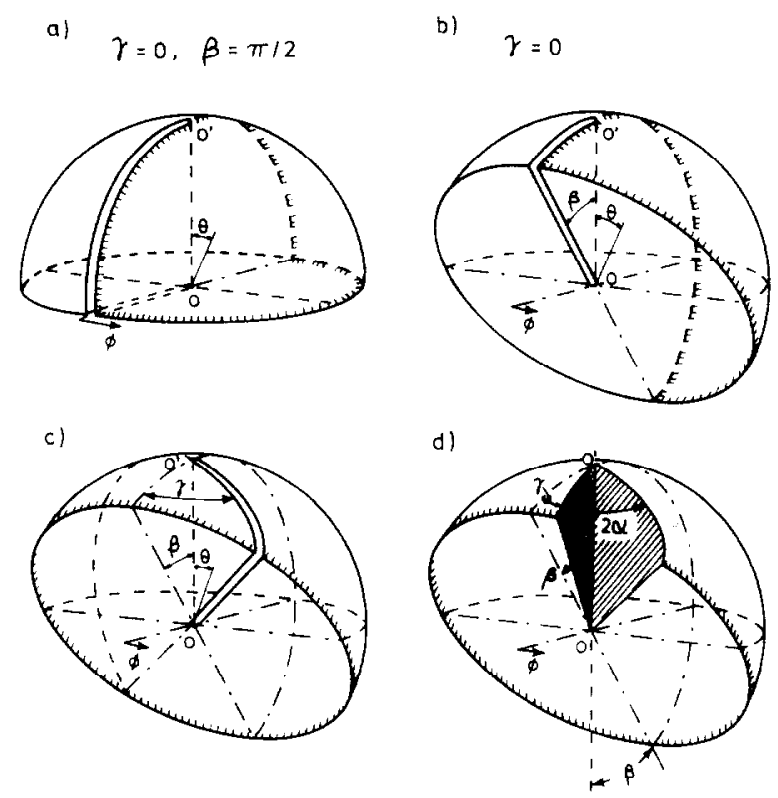

Fig. 1. Spherical coordinate system at termination of crack front edge $00^{\prime}$ at body surface. (a) orthogonal crack; (b) inclined edge; (c) inclined edge and inclined crack plane; (d) generally inclined notch. (The unit sphere is shown only to visualize the coordinates; the body is semi-infinite.) 
boundary conditions of symmetry (symmetric $v$, antisymmetric $\tau_{r \theta}$ and $\tau_{\phi \theta}$, i.e. $v=g=0$ and $\tau_{r \theta}=\tau_{\phi \theta}=0$ at $\theta=\pi / 2$ ). For the opening mode (Mode I) of such a crack, a crude finite difference solution based on a reduction to potential theory was given in [5] (yielding $\lambda=0.296$ for $\alpha=\pi / 2$ and all $\nu$ ), and a very accurate solution by means of singular integral equations has recently been obtained by Keer and Parihar[10] (yielding $\lambda=0.2966$ for $\alpha=\pi / 2$ ); the result of the present program $(\lambda=0.296)$, obtained by extrapolation from grids of up to 128 elements, agreed satisfactorily (see Figs. 3 and 4).

The antisymmetric mode for the same crack (characterized by $u \sin \theta=v \cos \theta$ and $\sigma_{\phi \phi}=0$ at $\phi=\pi$ ), also solved by Parihar and Keer[11], is irreducible to potential theory and depends on $\nu$, giving values $\lambda=0.2966$ and 0.3285 for $\nu=0.0$ and 0.25 . The present program (for the aforementioned grid) yielded $\lambda=0.296$ and 0.329 .

\section{NUMERICAL RESULTS FOR SURFACE CRACKS AND ANGLE OF PROPAGATING CRACK}

The main objective was to find the singular stress field near the point where a crack runs into the surface of the solid. This field corresponds to the smallest eigenvalue $\lambda$ (such that $\lambda>-1 / 2$ ). The solutions were obtained not only for cracks whose front edge is normal to the surface, but mainly for cracks whose front edge and plane form arbitrary angles $\beta$ and $\gamma$ with the solid surface (see Figs. 1a-c). Mode I for orthogonal crack edge has been solved analytically by Benthem [1], but no analytical solution is available for the oblique cases. (For all cases it has been assumed that $p=0$.)

The domain in $(\theta, \phi)$ plane which is to be solved is depicted in Fig. 2(a), along with the densest finite element grid used (a $12 \times 24$ grid, giving a system of 975 equations of band width 89 ). In the special cases which exhibit symmetry with respect to the crack plane $(\gamma=0)$, one needs only one half of the grid, and the densest grid used is shown in Fig. 2(b). Otherwise (for $\gamma \neq 0$ ) the whole domain $(0 \leq \phi \leq 2 \pi)$ must be solved. The grids have been generated automatically. The vertical grid lines are the meridians of the spherical coordinate system. The bottom

a)

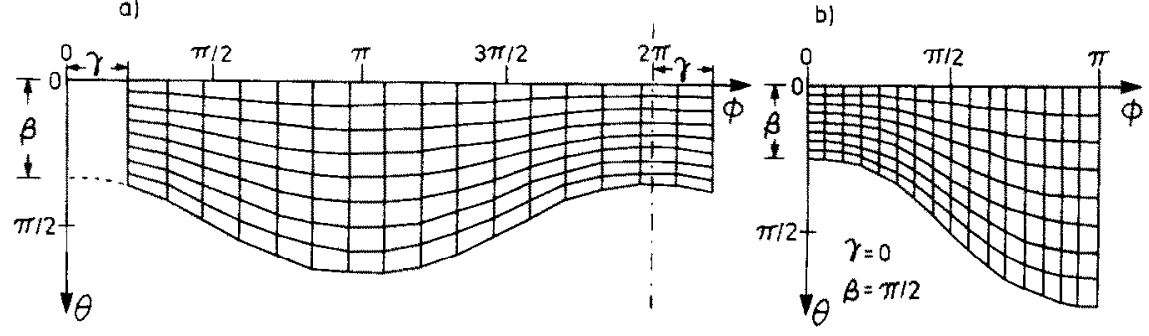

Fig. 2. Finite element grids used for inclined cracks. (a) both edge and crack plane inclined; (b) inclined edge, normal crack plane.
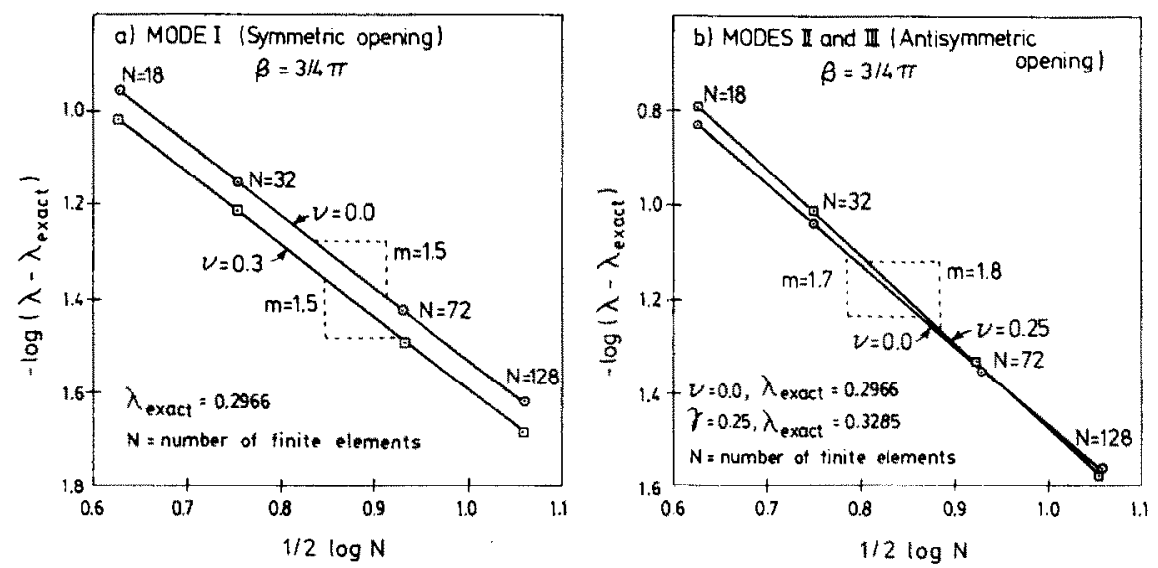

Fig. 3. Finite element convergence pattern for right angle corner at front edge of planar crack inside elastic body. (a) Mode I; (b) Modes II and III. 

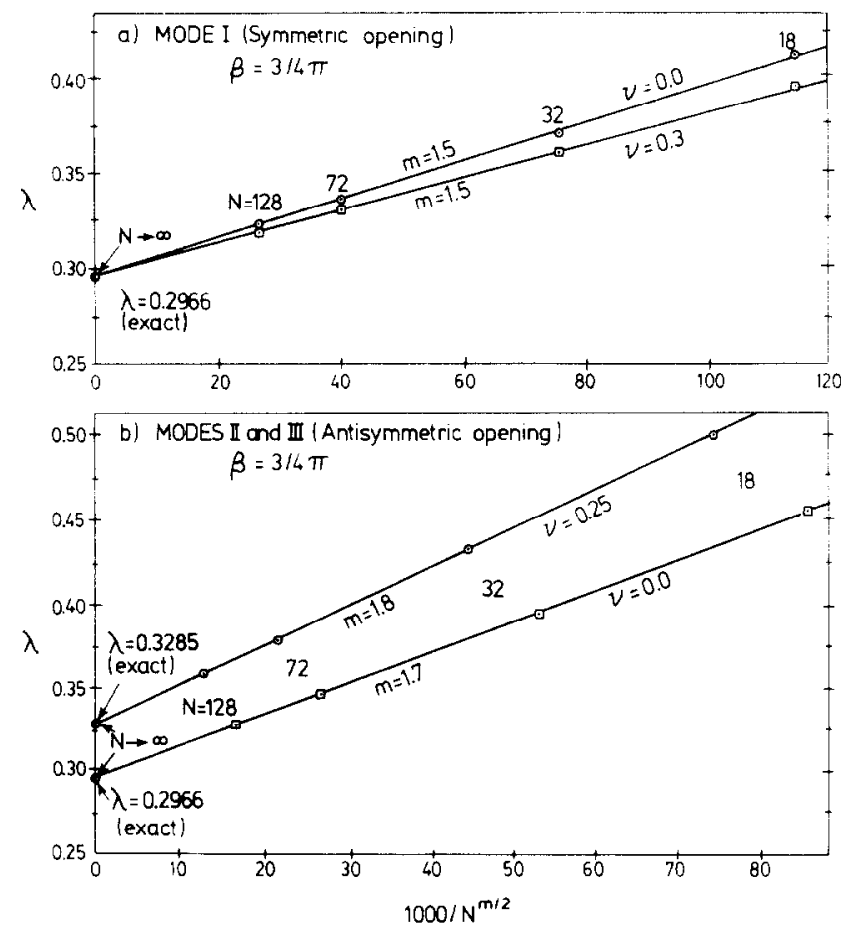

Fig. 4. Extrapolation of numerical results to $N \rightarrow \infty$ for the case in Fig. 3.

boundary of the domain is a great circle of the unit sphere which is given by the equation

$$
\theta=\theta_{b}=\arctan \left(\frac{\tan \beta}{\cos \phi}\right) ; \quad \text { if } \theta<0 \text { then } \theta \leftarrow \theta+\pi
$$

The nodal subdivision of each meridian was uniform.

Grids with nonuniform subdivision of the meridian, refined toward the pole, were also tried (Fig. 5) (for $\beta=\pi / 2, \gamma=0$ ). Although the results for the same maximum size of element were better, the results for the same total number of equations were no better than those for uniform subdivisions (see also Ref. [4]). So, nonuniform subdivisions would be ineffective.

The stress boundary conditions on the crack surfaces $(\phi=\gamma$ and $\phi=\gamma+2 \pi)$ and on the half-space surface $\left(\theta=\theta_{b}\right)$ are automatically satisfied by the finite element equations (which brings about a tremendous simplification compared to the finite difference method). The boundary conditions at $\theta=0$ (pole, top side of the domain in Fig. 2) are irrelevant and none have been imposed. To implement a statically determinate support of the body, one node may be fixed in the $\phi$ direction to prevent rotation about the polar axis. However, since rotation implies $\lambda=1$, the support is normally not necessary unless $\lambda=1$.

If the crack plane is normal to the body surface $(\gamma=0)$, only half the domain from Fig. 2(a) need be solved (see Fig. 2b) because there exists symmetry with respect to the plane $\phi=\pi$. In case of the symmetric (Mode I) crack opening, one must impose for all nodes at $\phi=\pi$ the condition $w=0$, i.e. $h=0$, while the symmetry conditions for stresses, namely $\tau_{\phi r}=\tau_{\phi \theta}=0$, are automatically satisfied by the finite element method as natural boundary conditions.

In case of antisymmetric crack openings the question of proper antisymmetry conditions is more complicated, and it appears that Modes II and III cannot exist separately at the surface point (which was first suggested by L. M. Keer of Northwestern University in a private communication). Indeed, it is impossible imagining the conditions of zero stress at the half-space surface to be satisfied by a displacement field that exhibits either Mode II or Mode III antisymmetry. The calculations confirmed this; i.e. when the full domain $\phi \in(0,2 \pi)$ was used and Mode II antisymmetric displacements were enforced in two symmetrically located nodes at the crack surface $(u=1$ at $\phi=0$ and $u=-1$ at $\phi=2 \pi$, both at $\theta=\pi / 2)$, the $v$ displacements at $\theta=\pi / 2$ were found to be non-zero and exhibit perfect antisymmetry about 


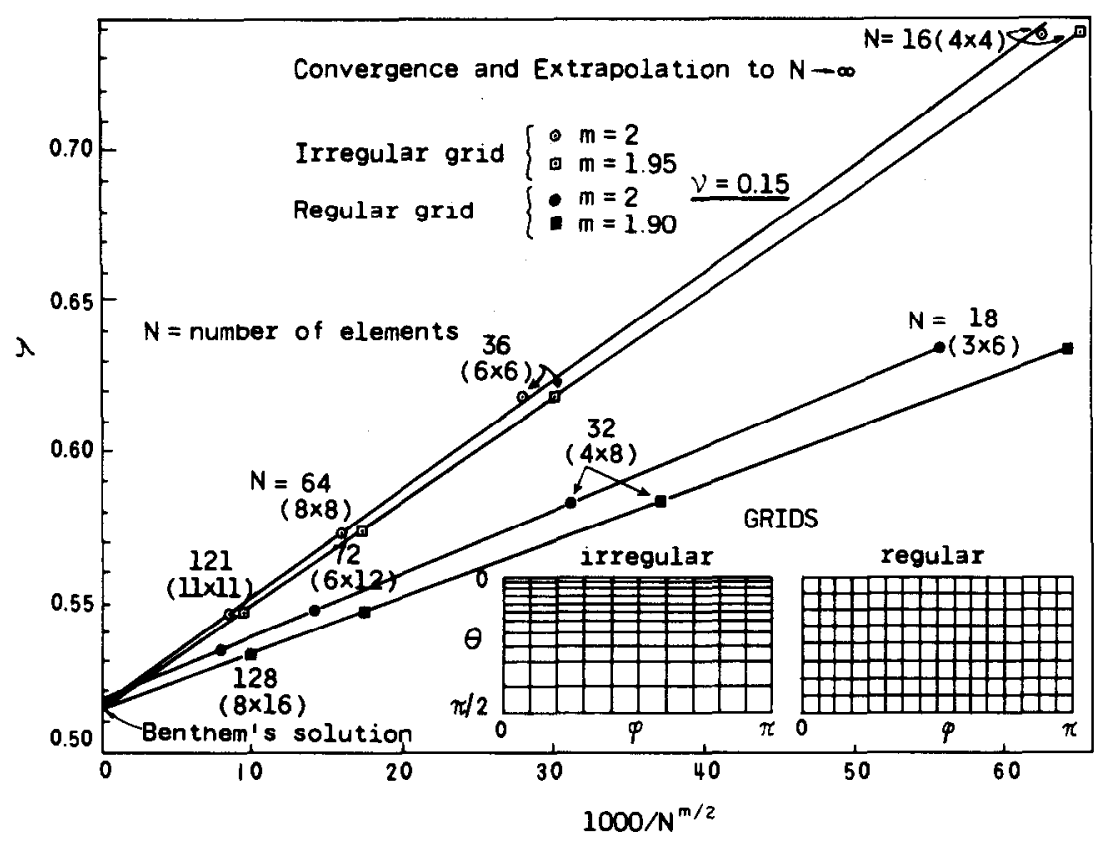

Fig. 5. Extrapolation of numerical results for orthogonal crack $(p=0)$ with grid refinement for Poisson's ratio $\nu=0.15$

$\phi=\pi$, which is characteristic of Mode III. Thus, one may impose at $\phi=\pi$ either $u \sin \theta-$ $v \cos \theta=0$ (Mode II-type condition), or $u \cos \theta-v \sin \theta=0$ (Mode III-type condition) or any linear combination of these two conditions, among which the simplest choice is $u=v=0$. The exponent $\lambda$ in either case is the same, and because it belongs to a combination of two modes, $\lambda$ is a double root. The antisymmetry condition for stress in both modes is $\sigma_{\phi \phi}=0$ at $\phi=\pi$, and it is again automatically satisfied by the finite element method as a natural boundary condition.

An idea of the accuracy and convergence was given in Ref. [4]. Eigenvalue $\lambda$ was solved for $\nu=0$, in which case the exact value is known to be $\lambda=0.5$. Grids of increasing numbers of identical rectangular finite elements on the $(\theta, \phi)$ plane were used; $N=18,32,72$ and 128 , corresponding to subdivisions $3 \times 6,4 \times 8,6 \times 12$ and $8 \times 16$ in the $\theta$ and $\phi$ directions. For the finest grid, which gives a system of 459 equations of bandwidth 65 , the result was $\lambda=0.5097$, which is still $1.9 \%$ in error. Finer grids would necessitate lavish computer funding. Higher order finite elements would no doubt improve accuracy drastically. However, this appeared to be unnecessary, since the convergence pattern can be exploited to greatly improve the accuracy, provided that the grids for various subdivisions are all similar, generated according to the same rule [4]. The numerical calculations indicated that the convergence is monotonous and of power type. In the case of $\nu=0$, exponent $p=0$ is absent from the exact solution, and so one may set $p=1 / 2$ on the basis of the previous discussion; then $f, g$ and $h$ have no gradient singularity, and a quadratic convergence should take place. This means that the plot of $\log (\lambda-1 / 2)$ vs $\log V(N)$ (see Fig. 2 of Ref.[4]) should be a straight line and should have the slope $m=2$. Numerical results confirmed this to be true [4]. The plots of $\lambda$ vs $1000 / N$ should also be straight lines, and they indeed are, which allows extrapolation to $N \rightarrow \infty$. This extrapolation provided $\lambda=0.500$ with an error not exceeding 0.001 .

Subsequently, the case of orthogonal crack edge was solved for various values $\boldsymbol{\nu}>\mathbf{0}$. For cracks of Mode I as well as Modes II and III, the results are given for various grid subdivisions of the half domain (half in view of symmetry) in Figs. 6 and 7 . In these cases, the exponent $p=0$ is known to be present, together with $p=1 / 2$. So, $p=0$ had to be introduced, causing functions $f, g$ and $h$ to exhibit gradient singularities. Therefore, quadratic convergence could not be expected. Nevertheless, it was assumed that the convergence is at least of power type $O\left(h^{m}\right)$, with $m<2$. To determine $m$, the results of $\lambda$ for various grids were plotted vs $1000 / N^{m / 2}$ for various chosen values of $m$, and each of them was fitted by a straight line (computationally). Then, the value of $m$ that gives the best straight-line fit (the smallest sum of square deviations) was determined in the program (Fig. 6). The resulting value of $m$ represents the rate of 


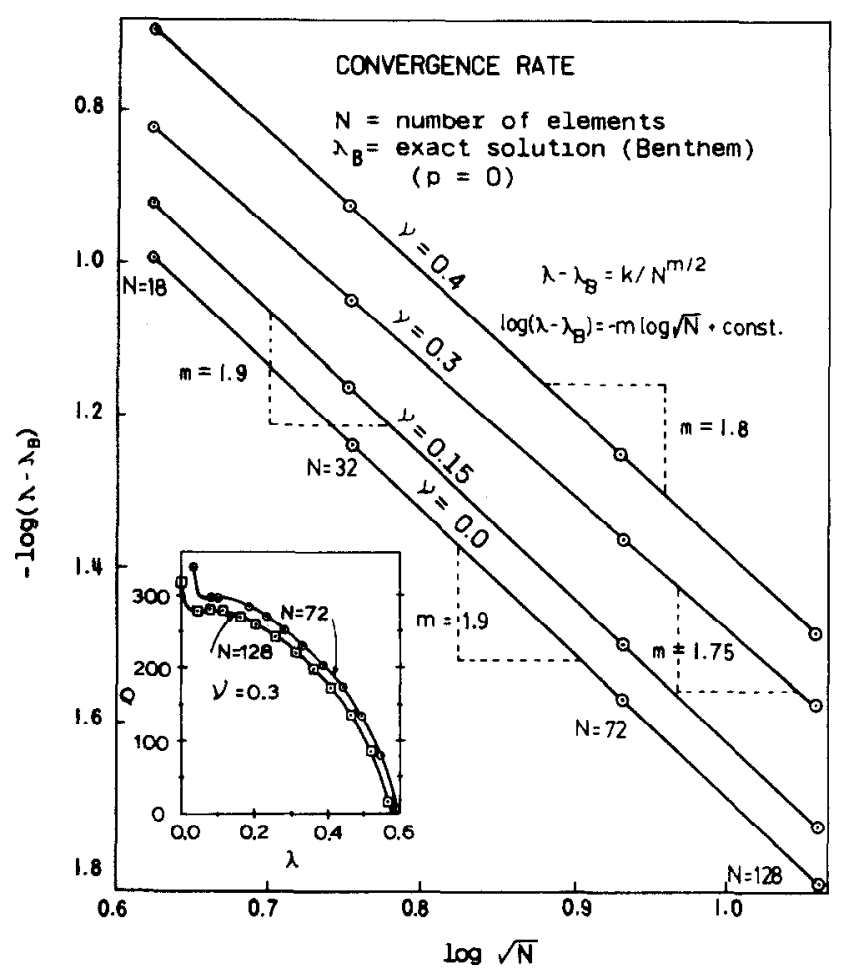

Fig. 6. Power-type convergence $(p=0)$ for orthogonal crack for various Poisson ratios. (Exact $\lambda$ after Benthem[1] are: for $\nu=0: \lambda=0.5$; for $\nu=0.15: \lambda=0.5164$; for $\nu=0.3: \lambda=0.5477$; for $\nu=0.4: \lambda=0.5868$.)

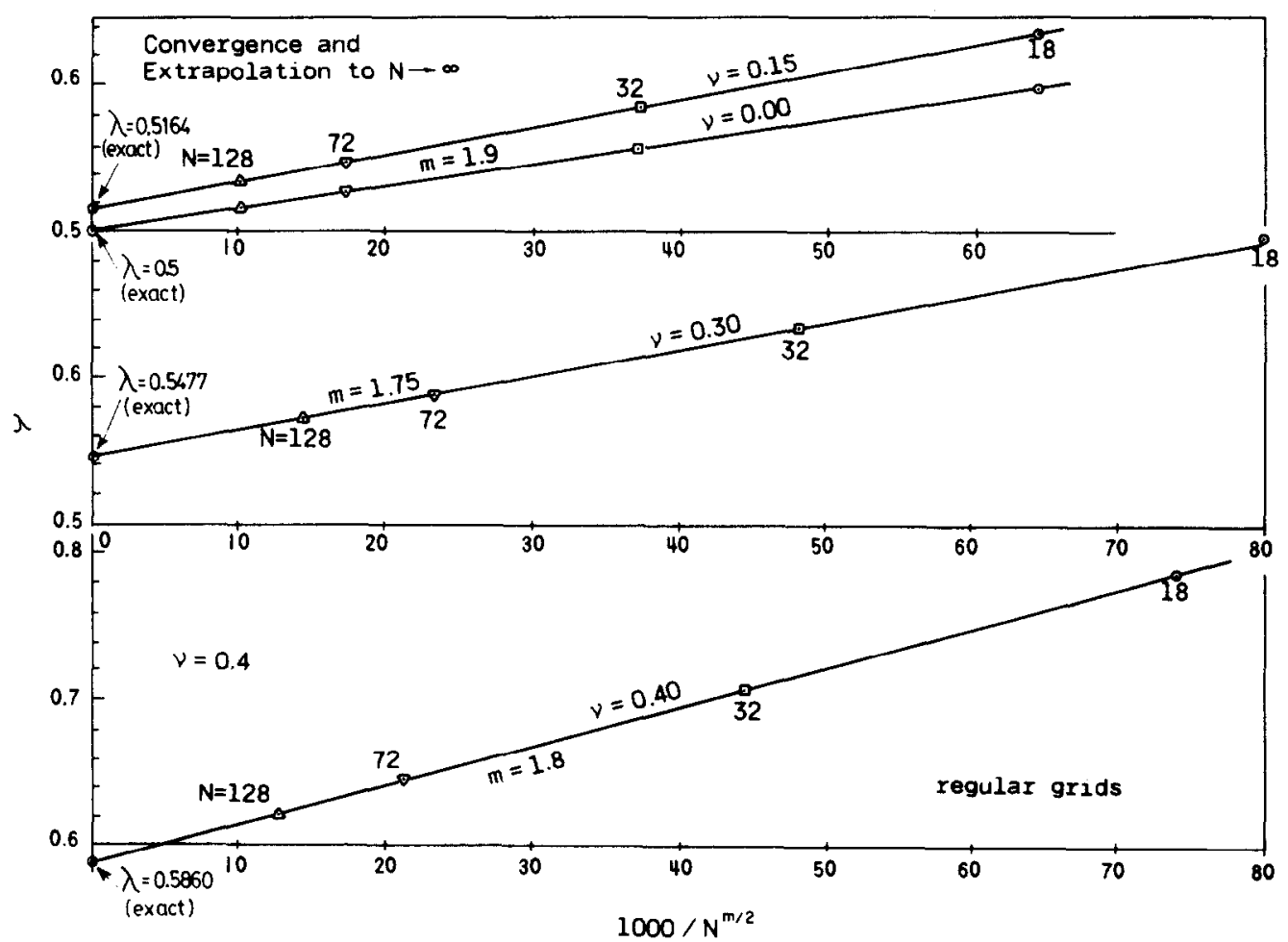

Fig. 7. Extrapolation of numerical results from Fig. 6 to $N=\infty$. 
convergence, which was always quite close to quadratic, with $m$ varying between 1.7 and 1.9. For oblique cracks, $m$ varied between 1.3 and 1.8 .

The extrapolations to $N \rightarrow \infty$ obtained with the optimum $m$-values are plotted in Figs. 8(a) and $8(\mathrm{~b})$. They agree with Benthem's results for Mode I cracks [1] within about $0.4 \%$ for all $\nu$ between 0 and $0.48[4]$. This is a strong mutual confirmation for the accuracy and correctness of the Benthem's solution as well as the present solution. No solution seems to exist in the literature for Modes II and III (Fig. 8b).

Kawai obtained for this problem $\lambda=0.3$ for $\nu=0$ as the lowest root [2]. This disagrees with Benthem [1] as well as our solution. The r.h.s. $Q$ of one of the equations whose vanishing is used to find the root (see method $B$ in Ref.[5]) is shown within Fig. 6 to demonstrate that the plot is very smooth and no eigenvalue $\lambda$ near 0.3 could have been missed.

According to eqn (20), a crack which propagates or for which propagation is imminent must exhibit $\lambda=1 / 2$ (the exponent being assumed to be real if there are no dissimilar materials). This case is of the greatest practical interest by far. For the orthogonal crack edge, propagation is obviously possible only if $\nu=0$. Therefore, we must ask whether there exist inclinations $\beta$ of
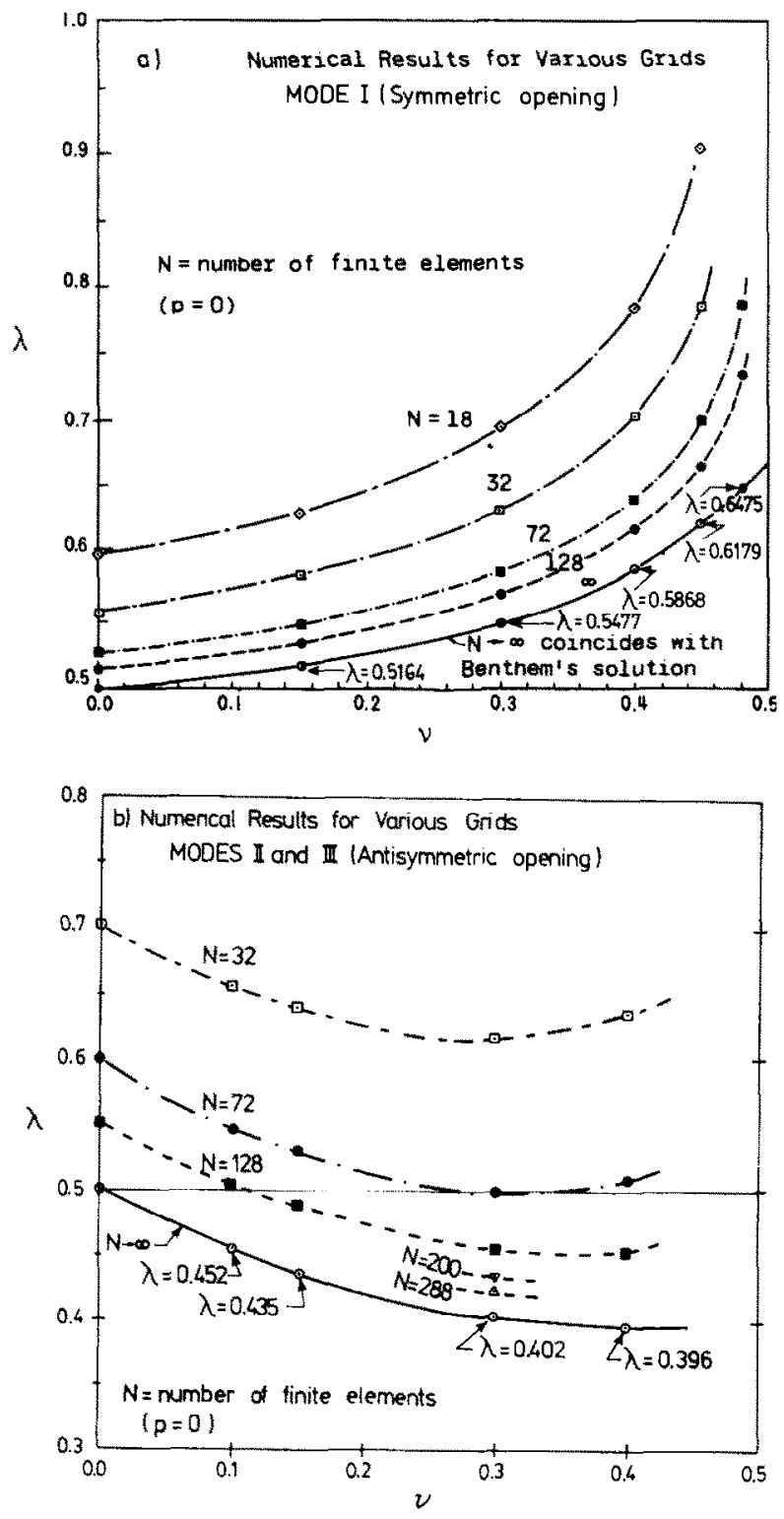

Fig. 8. Numerical results for orthogonal crack ( $p=0$ ). (a) Mode I (values from Figs. 6 and 7); (b) Modes II and III. 
the crack front edge and $y$ of the crack plane for which $\lambda=1 / 2$. Calculations indicated that indeed for each chosen $\gamma$ there exist $\beta$-values for which $\lambda=1 / 2$; see Figs. 9 to 11 .

To obtain accurate values of $\beta$ for each chosen $\gamma$, the approach to the eigenvalue problem was modified by treating $k_{i j}$ as a function of $\beta$ rather than $\lambda$ (because $\lambda$ is fixed); i.e. eqn (11) was treated as [4]:

$$
\sum_{i=1}^{M} k_{i j}(\beta) X_{j}=0 \quad(i=1,2, \ldots M)
$$

The eigenvalue search routine [5] based on Newton method was converted to a search for $\beta$ instead of $\lambda$.

First the results were obtained for cracks whose plane is normal to body surface $(\gamma=0)[4]$, and it was rather interesting to observe that the values of $\beta$ for the symmetric opening (Mode I) and for the antisymmetric opening (Modes II and III) were different; the former case gives an obtuse angle $\left(\beta>90^{\circ}\right)$, i.e. the surface point trails behind the interior crack edge, and the latter case gives an acute angle $\left(\beta<90^{\circ}\right)$, i.e. the surface point moves ahead of the interior crack edge. This has an important physical consequence: at the terminal point a combined mode propagation is impossible, i.e. the crack assumes such a shape that its surface terminal point propagates either with a symmetric opening (Mode I) or with an antisymmetric opening (Modes II and III), but not both combined.
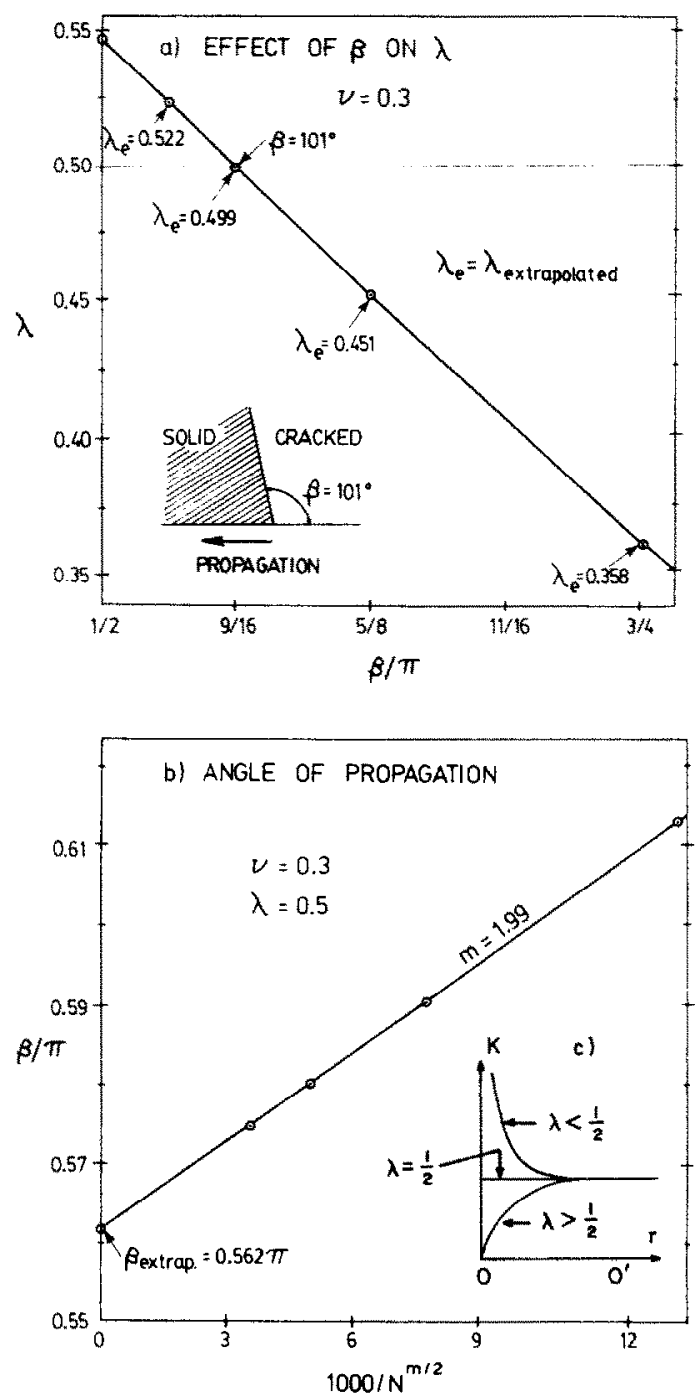

Fig. 9. Dependence of singularity exponent $\lambda$ upon front edge angle for normal crack plane (for $\nu=0.3$ ). 


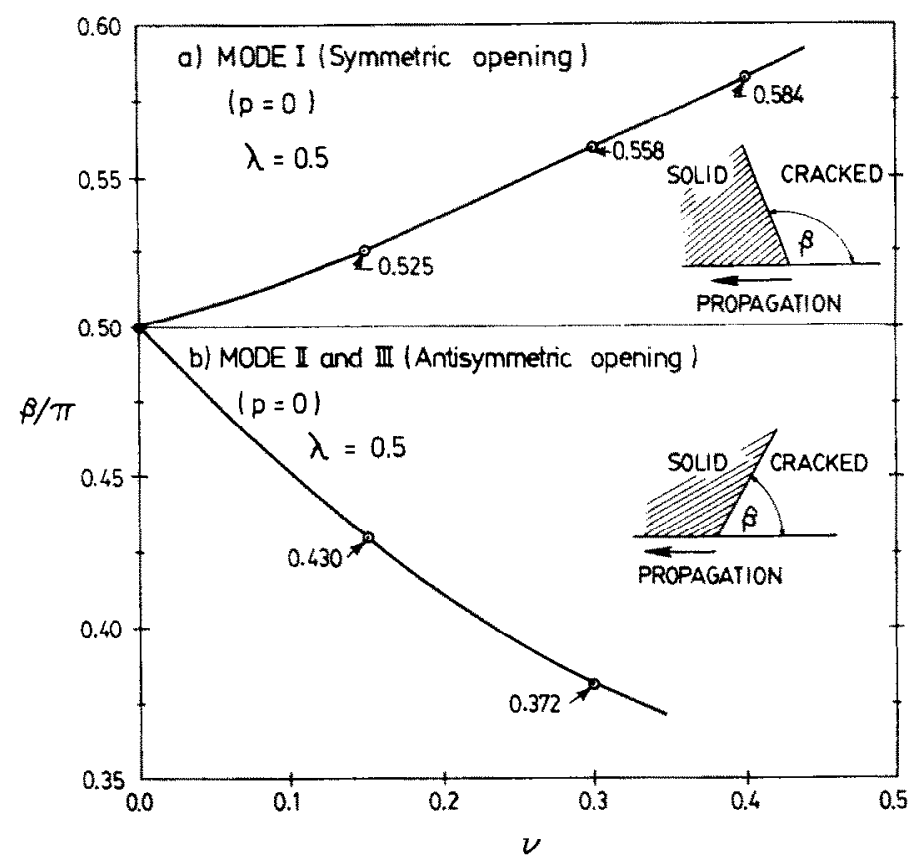

Fig. 10. Dependence of crack front edge angles $\beta$ of a propagating crack upon Poisson ratio $\nu$. (a) Mode I; (b) Modes II and III (normal crack plane).

As the crack plane becomes inclined $(\gamma \neq 0)$, the $\beta$-values for $\operatorname{Re}(\lambda)=(1 / 2)$ vary as a function of $\gamma$, as is seen from the numerical results in Fig. 11. In these cases it is no longer possible to distinguish between symmetric (Mode I) and antisymmetric (Modes II and III) openings, for there is no geometrical symmetry. For each of the two $\beta$-values, there exists at point 0 a certain limiting ratio $K_{1}: K_{2}: K_{3}$ of the stress intensity factors for Modes I, II and III, and no other ratios are possible. So, for cracks of inclined plane the propagation at the surface point takes place always in a combination of all three modes. Conversely, for a given ratio $K_{1}: K_{2}: K_{3}$ one can generally find the angles $\beta$ and $\gamma$ which must get established at the surface point.

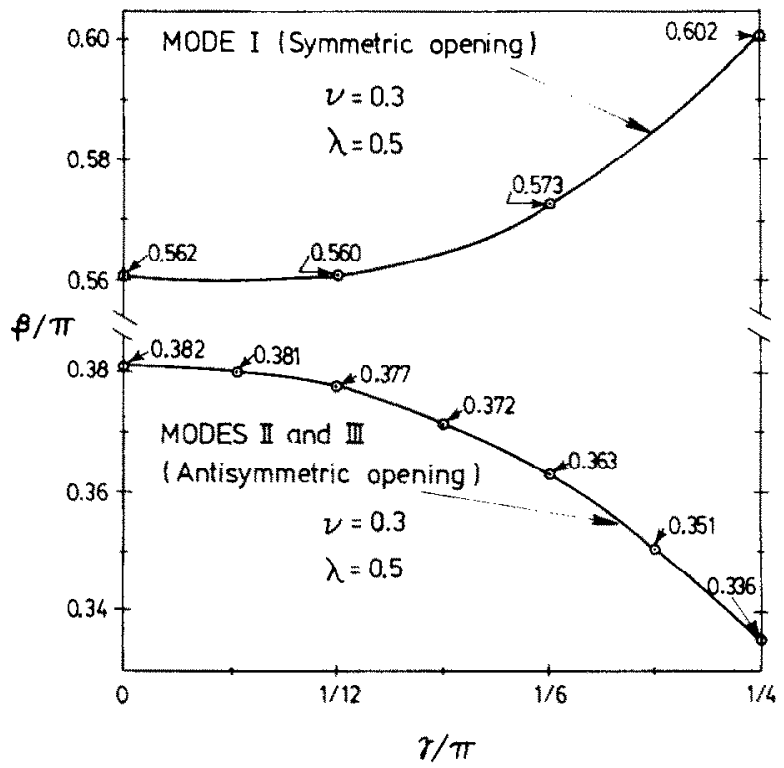

Fig. 11. Dependence of crack front edge angles $\beta$ of a propagating crack upon crack plane angle. $\gamma_{+}$(a) Mode I; (b) Modes II and III (for $\nu=0.3$ ). 
Some recent experimental results (pointed out to the writers by G. Sendeckyj of Flight Dynamics Laboratory, Dayton, Ohio) allow a check. These are the fatigue loading fracture tests made by Bell and Feeney[21] (to whom the writers are obliged for making available their results). Their photographs, reproduced in Figs. 12 and 13, show the crack arrest marks observed in fatigue Mode I fracture tests of aluminum alloy and titanium alloy specimens. According to materials handbooks, $\nu$ is about 0.33 or 0.32 , respectively, for which our solution (Fig. 10) gives $\beta \approx 102^{\circ}$ for both materials. These angles are plotted and compared in Figs. 12 and 13. Qualitatively, the observed trend agrees in that the surface point trails behind the interior crack edge (i.e. $\beta>90^{\circ}$ ) rather than moving ahead. The numerical value does not agree closely, but considering that some small-scale yielding and inelastic strain reversals occur in the actual tests, and that the plastic "shear-lip" phenomenon can alone also cause $\beta>90^{\circ}$, the comparison cannot be qualified as poor.

Some cases of notches terminating at the surface have also been run. The results for $\beta=\pi / 2$ (orthogonal edge), and $\nu=-\alpha$ (symmetry) are shown in Fig. 14.

\section{Problems with two-material interfaces}

In case of surface singularity of an interface crack or intersection of the crack front edge with a two-material interface, $\lambda$ must be expected to be complex, and so must be $K_{i j}$ and $X_{j}$. Conversion of the program to complex arithmetic[20] is straightforward and is achieved by COMPLEX type declarations of proper FORTRAN variables. Some difficulties were, however, caused by the need for an equation solving subroutine for complex banded nonsymmetric matrices. Such a subroutine has not been available in standard software packages, and so it had to be developed (and is listed in Ref.[20]). Furthermore, the iterative root search, described below eqn (12), had to be generalized so as to iterate for two simultaneous conditions: $\operatorname{Re}\left(R_{k}\right)=0$ and $\operatorname{Im}\left(R_{k}\right)=0$. Of these, the second one always converged much faster than the first one. However, it was necessary to start the iterations with a rather good initial estimate of $\lambda$. For this purpose, the plane of $\operatorname{Re}(\lambda)$ and $\operatorname{Im}(\lambda)$ was first scanned to find where the points of minimum value of $\left(R_{k}\right)^{2}$ lie, and those were then taken as the starting values for the iterations. As for the extrapolation based on meshes of different size, it was used only for $\operatorname{Re}(\lambda)$ and not for $\operatorname{Im}(\lambda)$ where no systematic convergence was found to exist.

The program was checked for giving correct numerical results in various check cases. For example, the three-dimensional case of a rigid stamp of wedge shape of angle $0.2886 \pi$, bonded to a homogeneous elastic halfspace surface of $\nu=0.3$ and subjected to a displacement parallel to the surface gives $\lambda=0.2475 \pm 0.0409 i$ (obtained analytically by $L$. M. Keer), while the program yielded $\operatorname{Re}(\lambda)=0.2405$ (for $N \rightarrow \infty$ ) and $\operatorname{Im}(\lambda)=0.0451$ (for $N=128$ ). The plane strain problem of an interface crack between two materials which both have $\nu=0.3$ and have a 40:1 ratio of $E$ has the solution $\lambda=0.5 \pm 0.0878 i[22]$, and the present program yielded $\operatorname{Re}(\lambda)=0.498$ $(N \rightarrow \infty)$ and $\operatorname{Im}(\lambda)= \pm 0.087 i(N=124)$.

When $\nu=0$ for both materials, the plane strain solution[22] also applies for our surface singularity with orthogonal crack edge; for a $2: 1$ ratio of $E$ this gives $\lambda=0.5 \pm 0.0535 i$ whereas the program yielded $\operatorname{Im}(\lambda)=0.0514$ for $N=128$. For the same case but $\nu>0$ no solutions have been given before; our program gives $\operatorname{Im}(\lambda)= \pm 0.0399(N=128)$ for $\nu=0.05$ and \pm 0.006 for $\nu=0.3$, and we see that $\operatorname{Im}(\lambda)$ decreases with increasing $\nu$. For $\nu=0.3$ and a $30: 1$ ratio of $E$, the program indicates $\operatorname{Im}(\lambda)$ to be 0 or almost 0 .

The front edge angle of a propagating interface crack in a plane orthogonal to the halfspace surface must satisfy $\operatorname{Re}(\lambda)=0.5$ and also depends on the $E$-ratio of the two materials.

When an interior crack plane is orthogonal to two-material interface, the program indicates that a crack with a front edge orthogonal to the interface has $\lambda=0.545,0.521$ and 0.499 for $E$-ratios $1: 1,5: 0$ and $10: 0$ and $\nu=0.3(N \rightarrow \infty)$.

For complex $\lambda$, the displacements oscillate along the radial rays, which implies a physically impossible overlap of crack surfaces. However, for plane-strain Mode I crack the region of this oscillation is usually extremely small and beyond this small region the solution appears to be meaningful and accurate[23]. It is not clear whether this also applies for the foregoing three-dimensional singularities. A physically meaningful solution which takes into account the contact stresses on crack surfaces has recently been developed[23]; but its adaptation is 


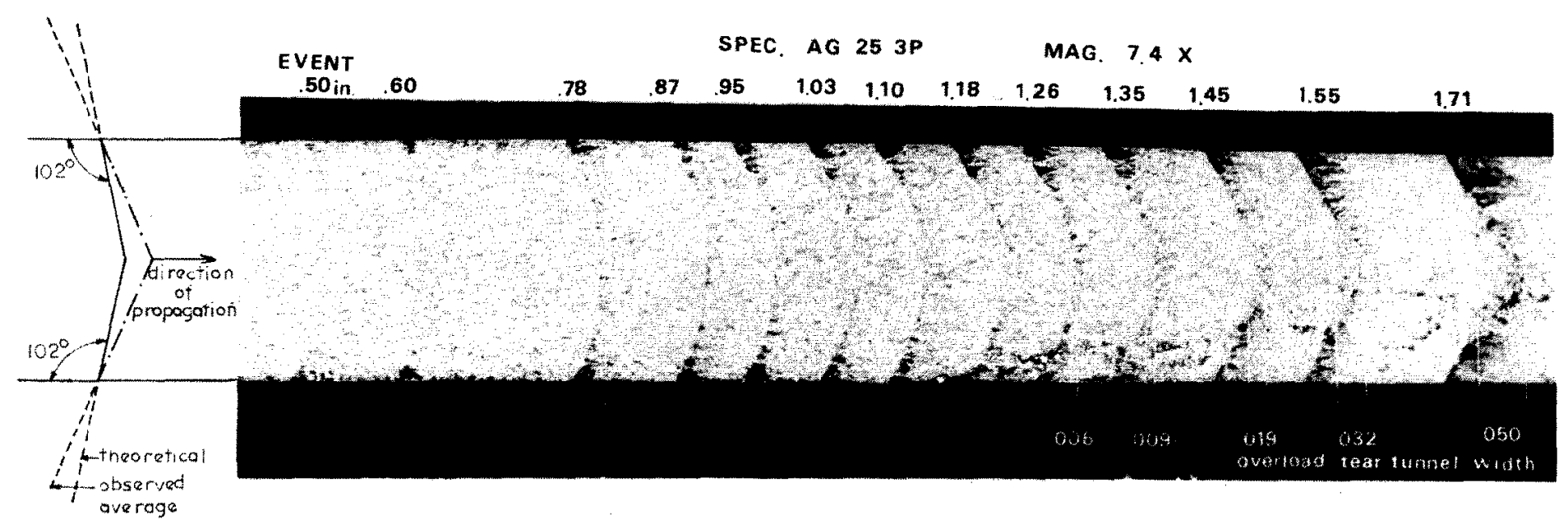

Fig. 12. View of fracture face showing fatigue arrest marks on aluminum alloy 2219-T581 $(\nu=0.33$, Mode I crack; sheet width $6.35 \mathrm{~mm}$ (1/4 in.); magnification 7.4 times; crack propagates to the right). (Reproduced from Fig. A3 on p. 160 of P. D. Bell and W. J. Feeney[21].) 


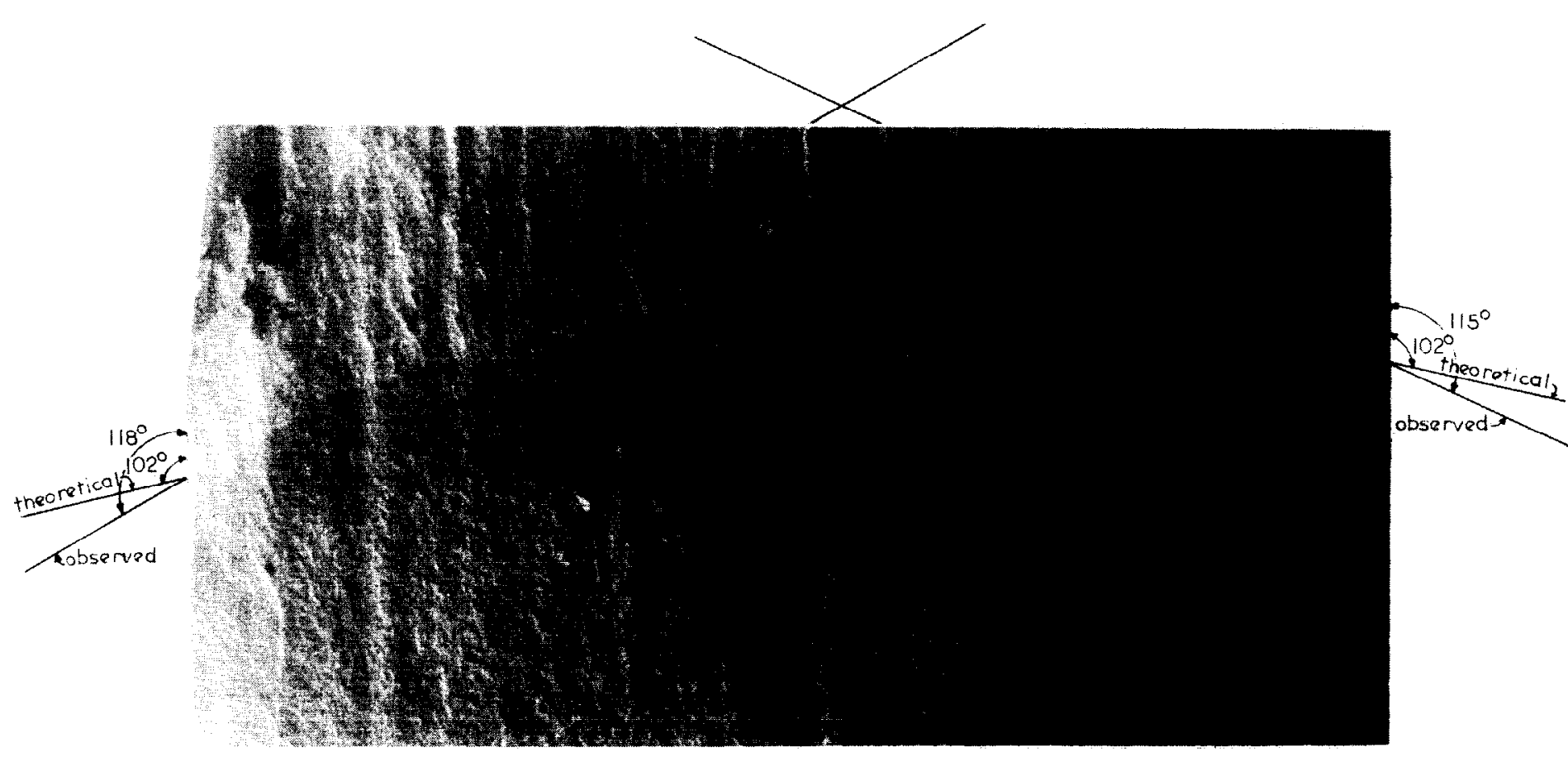

Fig. 13. View of fracture face showing crack arrest mark on titanium alloy Ti6 A1-4V ( $\nu=0.32$, Mode I crack; sheet width $6.35 \mathrm{~mm}(1 / 4 \mathrm{in}$.) magnification 33 times; crack propagates upwards). (Reproduced from Fig. A6 on p. 167 of P. D. Bell and W. J. Feeney[21].) 


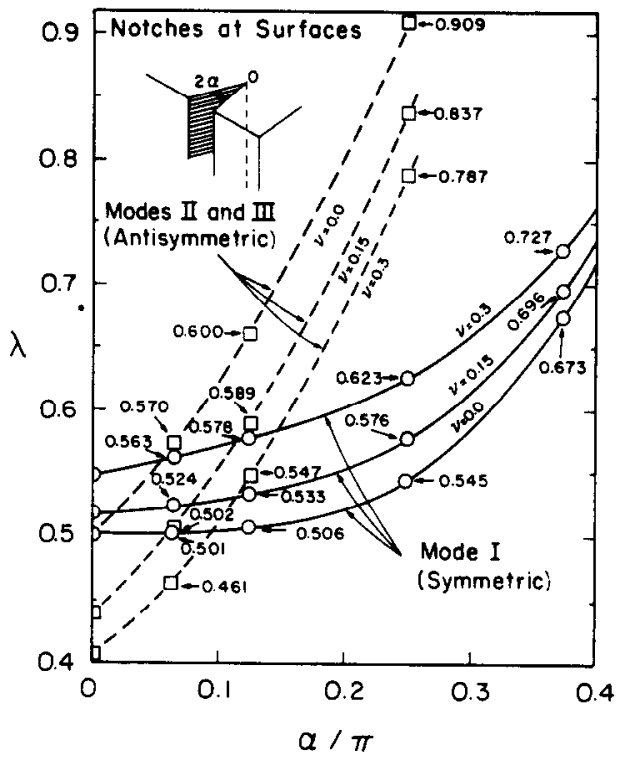

Fig. 14. Numerical results for surface termination of notches having orthogonal front edge $(N \rightarrow \infty)$.

beyond the scope of this study and we must content ourselves in the meantime with the less than perfect oscillating (complex) crack singularity. Note, though, that in many cases the complex singularity does not violate any contact conditions; e.g. when we have a notch of finite angle and a two-material interface emanates from the notch edge.

\section{CONCLUSIONS}

The finite element method in angular spherical coordinates provides a powerful general technique for determining three-dimensional elastic singular stress fields. The numerical results for cracks whose edge is normal to the surface are in close agreement with the analytical solution of Benthem. The front edge of propagating crack must terminate at the surface point obliquely, at a certain angle which is a function of the inclination of the crack plane and Poisson's ratio. For each combination of crack edge angle and crack plane angle only one certain ratio of the three stress-intensity factors is possible. If the crack plane is orthogonal, the surface point for Mode I opening trails behind the interior of the crack, while for Modes II and III the surface point moves ahead.

Acknowledgements-Grateful acknowledgment is due to the U.S. Air Force Office of Scientific Research for sponsoring the major part of this research under Grant No. AFOSR75-2859 to Northwestern University. Leon M. Keer, Professor at Northwestern University and co-director of the grant project, is thanked for his stimulating discussions and valuable suggestions during the progress of this work. The writers are also obliged to Jan P. Benthem, Professor at Delft University of Technology, for some very useful critical remarks and for clarifying the implications of his analytical solution for the $p$-value to be assumed, and to G. Sendeckyj, senior scientist at Air Force Flight Dynamics Laboratory, Dayton, Ohio, for helpful suggestions.

\section{REFERENCES}

1. J. P. Benthem, State of stress at the vertex of a quarter-infinite crack in a half-space. Int. J. Solids Structures 13, 479-492 (1977).

2. T. Kawai, Y. Fujitani and K. Kumagai, Analysis of Singularity at the Root of the Surface Crack Problem. Proc. Int. Conf. Fracture Mech. and Tech., Hong Kong, March 1977 (Edited by G. C. Sih and C. L. Chow), Vol. II, pp. 1157-1163.

3. E. S. Folias, On the three-dimensional theory of cracked plates. Trans. ASME (Ser. E), J. Appl. Mech. 42, 663-674 (1975).

4. Z. P. Bažant and L. F. Estenssoro, General Numerical Method for Three-Dimensional Singularities in Cracked or Notched Elastic Solids. Fracture 1977, Proc. 4th Int. Conf. Fracture, Univ. of Waterloo, Ontario, Canada, June 1977 (Edited by D. M. R. Taplin), Vol. 3, pp. 371-385.

5. Z. P. Bažant, Three-dimensional harmonic functions near termination or intersection or gradient singularity lines: A general numerical method. Int. J. Engng Sci. 12, 221-243 (1974).

6. Z. P. Bažant and L. M. Keer, Singularities of elastic stresses and of harmonic functions at conical notches and inclusions. Int. J. Solids Structures 10, 957-964 (1974).

7. J. D. Achenbach and Z. P. Bažant, Elastodynamic near-tip stress and displacement fields for rapidly propagating cracks in orthotropic materials. Trans. ASME (Ser. E), J. Appl. Mech. 42, 183-189 (1975). 
8. J. D. Achenbach, Z. P. Bažant and R. P. Khetan, Elastodynamic near-tip fields for a crack propagating along the interface of two orthotropic solids. Int. J. Engng Sci. 14, 811-818 (1976).

9. J. A. Morrison and J. A. Lewis, Charge singularity at the corner of a flat plate, SIAM, I. Appl. Math. 31, 233-250 (1976).

10. L. M. Keer and K. S. Parihar, Singularity at the corner of a wedge-shaped punch or crack. SIAM J. Appl. Math. 34, 297-302 (1978); for pyramidal notches see also Quart. Appl. Math. 401-405 (1977).

11. K. S. Parihar and L. M. Keer, Stress singularity at the corner of a wedge-shaped crack or inclusion. Trans. ASME, J. Appl. Mech. To be published (1979).

12. A. E. H. Love, A Treatise on the Mathematical Theory of Elasticity, 4th Edn. Dover, New York (1944).

13. M. Knein, Zur Theorie des Druckversuchs. Abhandlungen aus dem Aerodynamischen Instit an der Techn. Hochschule Aachen, Heft 7, 43-62 (1927).

14. M. L. Williams, Stress singularities resulting from various boundary conditions in angular corners of plates in extension. Trans. ASME, (Ser. E), J. Appl. Mech. 19, 526-528 (1952).

15. S. N. Karp and F. C. Karal, The elastic-field behavior in the neighborhood of a crack of arbitrary angle. Commun. Pure Appl. Math. 15, 413-421 (1962).

16. J. L. Swedlow and M. E. Karabin, A Case of Elasto-Plastic Flow Using a New Special Element. Fracture 1977, Proc. 4th Int. Conf. Fracture, Univ. of Waterloo, Ontario, Canada, June 1977 (Edited by D. M. R. Taplin), Vol. 1, 117-131.

17. W. G. Strang and G. J. Fix, An Analysis of the Finite Element Method. Prentice-Hall, Englewoud Cliffs, New Jersey (1973).

18. Z. P. Bažant and M. Christensen, Analogy between micropolar continuum and grid frameworks under initial stress. Int. J. Solids Structures 8, 327-346 (1972).

19. J. F. Knott, Fundamentals of Fracture Mechanics. Butterworth, London (1973).

20. Z. P. Bažant and L. F. Estenssoro, The Surface Singularity of a Crack. Structural Engineering Report No. 78-8/480 (to Air Force Office of Scientific Research), Northwestern University, Evanston, Illinois, Aug. 1978.

21. P. D. Bell and W. J. Feeney, Fractographic Evaluation of Fracture Specimens. Tech. Rep. AFFDL-TR-75-152 (to Air Force Flight Dynamics Laboratory, Wright-Patterson AFB, Dayton, Ohio), Grumman Aernspace Corporation, Bethpage, N.Y. (1976).

22. F. Erdogan and G. Gupta, Layered composites with an interface flaw. Int. J. Solids Structures 7, 1089-1107 (1971).

23. M. Comninou, The interface crack. Trans. ASME (Ser. E), J. Appl. Mech. 44, 631-636 (1977).

24. O. C. Zienkiewicz, The Finite Element Method in Engineering Science. McGraw-Hill, London (1971).

25. Z. P. Bažant, J. L. Glazik and J. D. Achenbach, Elastodynamic fields near running cracks by finite elements. Comput. Structures 8, 193-198 (1978)

\section{APPENDIX A}

Proof of the variational principle

Since the derivation of the basic variational equation (eqn 7) involves an unorthodox step-integration by parts with respect to only one coordinate, $r$, a step which destroys the symmetry of the functional, it is appropriate to check that the Euler equations associated with the variational equation are the correct differential equations and natural boundary conditions for functions $F, G$ and $H$. To deduce the Euler equations, one may apply Green's integral theorem in the plane $(\theta, \phi)$ with $\theta$ and $\phi$ regarded as cartesian coordinates, so as to get rid of derivatives of $\delta F, \delta G$ and $\delta H$. By this procedure, eqn (7) for $C=$ const. may be brought to the form

$$
\iint_{A}\left(X, \delta F+X_{\theta} \delta F+X_{\phi} \delta H\right) \sin \theta \mathrm{d} \theta \mathrm{d} \phi-\int_{s}\left(p, \delta F+p_{\theta} \delta G+p_{\phi} \delta H\right) \mathrm{d} s=0
$$

in which

$$
\begin{aligned}
X_{r}= & (Q+2)(\lambda-1)\left(\lambda F+F+G_{\theta}+G \cot \theta+\frac{1}{\sin \theta} H_{\phi}\right)-\left[(\lambda+1) G_{\theta}-F_{\theta \theta}\right] \\
& -\cot \theta\left[(\lambda+1) G-F_{\theta}\right]+\frac{1}{\sin \theta}\left(\frac{1}{\sin \theta} F_{\phi \phi}-H_{\phi}-\lambda H_{\phi}\right)=0 \\
X_{\theta}= & (Q+2)\left(\lambda F_{\theta}+2 F_{\theta}+G_{\theta \theta}+G_{\theta} \cot \theta-\frac{1}{\sin ^{2} \theta} G+\frac{1}{\sin \theta} H_{\theta \phi}-\frac{\cos \theta}{\sin ^{2} \theta} H_{\phi}\right) \\
& -\frac{1}{\sin \theta}\left(H_{\theta \phi}+H_{\phi} \cot \theta-\frac{1}{\sin \theta} G_{\phi \phi}\right)+\lambda\left[(\lambda+1) G-F_{\theta}\right]=0 \\
X_{\phi}= & \frac{1}{\sin \theta}(Q+2)\left(\lambda F_{\phi}+2 F_{\phi}+G_{\theta \phi}+G_{\phi} \cot \theta+\frac{1}{\sin \theta} H_{\phi \phi}\right)-\lambda\left(\frac{1}{\sin \theta} F_{\phi}-H-\lambda H\right) \\
+ & \left(H_{\theta \theta}+H_{\theta} \cot \theta-\frac{1}{\sin ^{2} \theta} H+\frac{\cos \theta}{\sin ^{2} \theta} G_{\phi}-\frac{1}{\sin \theta} G_{\theta \phi}\right)=0
\end{aligned}
$$

and

with

$$
\begin{gathered}
p_{r}=s_{r \theta} n_{\theta} \sin \theta+s_{r \phi} n_{\phi}=0 \\
p_{\theta}=s_{\theta \theta} n_{\theta} \sin \theta+s_{\theta \phi} n_{\phi}=0 \\
p_{\phi}=s_{\theta \phi} n_{\theta} \sin \theta+s_{\phi \phi} n_{\phi}=0
\end{gathered}
$$

$$
\begin{aligned}
& s_{r \theta}=\frac{1}{2 \mu r^{\lambda-1}} \sigma_{r \theta}=\lambda G-G+F_{\theta} \\
& s_{\theta \theta}=\frac{1}{\mu r^{\lambda-1}} \sigma_{\theta \theta}=Q\left(\lambda F+2 F+G_{\theta}+G \cot \theta+\frac{1}{\sin \theta} H_{\phi}\right)+2\left(G_{\theta}+F\right)
\end{aligned}
$$




$$
\begin{aligned}
& s_{\theta \phi}=\frac{1}{2 \mu r^{\lambda-1}} \sigma_{\theta \phi}=H_{\theta}-H \cot \theta+\frac{1}{\sin \theta} G_{\phi} \\
& s_{r \phi}=\frac{1}{2 \mu r^{\lambda-1}} \sigma_{r \phi}=\frac{1}{\sin \theta} F_{\phi}+\lambda H-H \\
& s_{\phi \phi}=\frac{1}{\mu r^{\lambda-1}} \sigma_{\phi \phi}=Q\left(\lambda F+2 F+G_{\theta}+G \cot \theta+\frac{1}{\sin \theta} H_{\phi}\right)+2\left(\frac{1}{\sin \theta} H_{\phi}+G \cot \theta+F\right) .
\end{aligned}
$$

Here $s=$ length of the boundary curve of the region in the $(\theta, \phi)$ plane; $d s^{2}=\mathrm{d} \theta^{2}+\mathrm{d} \phi^{2} ;\left(n_{\theta}, n_{\phi}\right)$ represents the unit normal to the curve $s$ in $(\theta, \phi)$ plane in which $\theta$ and $\phi$ are regarded as cartesian coordinates, i.e. $n_{\theta}=\mathrm{d} \phi / \mathrm{d} s$ and $n_{\phi}=-\mathrm{d} \theta / \mathrm{d} s$ (derivatives along curve $s$ ); $\sigma_{r \theta}, \ldots \sigma_{\phi \phi}$ are the stress components in spherical coordinates; $\mu=$ shear modulus. Since eqn (A1) must hold for any kinematicaily admissible $\delta F, \delta G$ and $\delta H$, it implies that

$$
\begin{array}{lll}
X_{r}=0, & X_{\theta}=0, & \left.X_{\phi}=0 \text { (within } A\right) \\
p_{r}=0, & p_{\theta}=0, & \left.p_{\phi}=0 \text { (on } s\right) .
\end{array}
$$

It can now be checked that these equations are identical to those which arise when eqns (4) are substituted into the well-known[12] differential equations of equilibrium in terms of $u, v$ and $w$ :

$$
\begin{aligned}
& (\lambda+2 \mu) r \sin \theta \frac{\partial \Delta}{\partial r}-2 \mu\left\{\frac{\partial}{\partial \theta}\left(\bar{\omega}_{\phi} \sin \theta\right)-\frac{\partial \bar{\omega}_{\theta}}{\partial \phi}\right\}=0 \\
& (\lambda+2 \mu) \sin \theta \frac{\partial \Delta}{\partial \theta}-2 \mu\left\{\frac{\partial \tilde{\omega}}{\partial \phi}-\frac{\partial}{\partial r}\left(r \bar{\omega}_{\phi} \sin \theta\right)\right\}=0 \\
& (\lambda+2 \mu) \frac{1}{\sin \theta} \frac{\partial \Delta}{\partial \phi}-2 \mu\left\{\frac{\partial}{\partial r}\left(r \bar{\omega}_{\theta}\right)+\frac{\partial \tilde{\omega}_{r}}{\partial \theta}\right\}=0
\end{aligned}
$$

where $\lambda$ and $\mu$ are Lamé's constants, $\Delta$ is the cubical dilatation, and $\tilde{\omega}_{n} \tilde{\omega}_{\theta}$, and $\bar{\omega}_{\phi}$ are the components of rotation:

$$
\begin{aligned}
\Delta & =\frac{1}{r^{2} \sin \theta}\left\{\frac{\partial}{\partial r}\left(r^{2} u \sin \theta\right)+\frac{\partial}{\partial \theta}(r v \sin \theta)+\frac{\partial}{\partial \phi}(r w)\right\} \\
2 \bar{\omega}_{r} & =\frac{1}{r^{2} \sin \theta}\left\{\frac{\partial}{\partial \theta}(r w \sin \theta)-\frac{\partial}{\partial \phi}(r v)\right\} \\
2 \bar{\omega}_{\theta} & =\frac{1}{r \sin \theta}\left\{\frac{\partial u}{\partial \phi}-\frac{\partial}{\partial r}(r w \sin \theta)\right\} \\
2 \tilde{\omega}_{\phi} & =\frac{1}{r}\left\{\frac{\partial}{\partial r}(r v)-\frac{\partial u}{\partial \theta}\right\} .
\end{aligned}
$$

For boundary conditions, eqns (4) must be substituted into the stress boundary conditions in terms of $u, v$ and $w$, which are similar to eqns (A3) and are formulated in terms of stress expressions[12]:

$$
\begin{aligned}
& \sigma_{r r}=\lambda e+2 \mu \mu_{r}, \quad \sigma_{\theta \theta}=\lambda e+2 \mu\left(\frac{1}{r} v_{\theta}+\frac{1}{r} u\right), \\
& \sigma_{\phi \phi}=\lambda e+2 \mu\left(\frac{1}{r \sin \theta} w_{\phi}+\frac{1}{r} v \cot \theta+\frac{1}{r} u\right), \quad \sigma_{r \theta}=\mu\left(v_{r}-\frac{1}{r} v+\frac{1}{r} u_{\theta}\right), \\
& \sigma_{\theta \phi}=\mu\left(\frac{1}{r} w_{\theta}-\frac{1}{r} w \cot \theta+\frac{1}{r \sin \theta} v_{\phi}\right), \quad \sigma_{r \phi}=\mu\left(\frac{1}{r \sin \theta} u_{\phi}+w_{r}-\frac{1}{r} w\right)
\end{aligned}
$$

where $e=u_{r}+(2 / r) u+(1 / r) v_{\theta}+(1 / r) v \cot \theta+(1 / r \sin \theta) v_{\phi}$. This proves that eqn (8) is correct and that the natural boundary conditions are automatically implied, which is essential for allowing the use of the finite element method.

Instead of considering domain $A$ in plane $(\theta, \phi)$ and applying Green's theorem the same result can be obtained by applying Stoke's theorem and considering that domain $A$ is on a unit sphere, $r=1$. In this procedure, factor $\sin \theta$ must not be treated as part of the integrand in eqn (8), because the area element on the unit sphere is not $d \theta d \phi$, as in the $(\theta, \phi)$ plane, but $\sin \theta \mathrm{d} \theta \mathrm{d} \phi$.

In a preceding paper, the same variational equation (eqn 8 ), was derived by a reverse procedure starting from the differential equations (eqns A5). The present derivation from strain energy is simpler and more direct.

\section{APPENDIX B}

Formulation of finite element stiffness equations

For the reader's convenience, the derivation of finite element equations [4] will be sketched. According to eqn (9)

$$
\begin{array}{rlr}
\delta F=\sum_{i} F^{i} \delta X_{j}, & \delta G=\sum_{j} G^{i} \delta X_{j}, & \delta H=\sum_{i} H^{i} \delta X_{j} \\
\delta F_{\theta}=\sum_{i} F_{\theta}^{j} \delta X_{j}, & \delta G_{\theta}=\sum_{j} G_{\theta}^{j} \delta X_{i}, & \delta H_{\theta}=\sum_{i} H_{\theta}^{i} \delta X_{i} \\
\delta F_{\phi}=\sum_{i} F_{\phi} \delta X_{j}, & \delta G_{\phi}=\sum_{j} G_{\phi}^{i} \delta X_{j}, & \delta H_{\phi}=\sum_{j} H_{\phi}^{j} \delta X_{r}
\end{array}
$$


Substitution of these expressions as well as eqns (9) into eqn (6) furnishes

$$
\Phi_{F}=\sum_{T} \Phi_{F}^{i} X_{i}, \quad \Phi_{F}=\sum_{i} \Phi_{F}^{i} X_{p} \ldots \Phi_{H_{+}}=\sum \Phi_{H_{\psi}}^{i} X_{i}
$$

Moreover, substitution of equs (B1) and (B2) into the variational principle. (eqn 8), provides a discrete variational equation of the form

$$
\sum_{i=1}^{4}\left[\sum_{i=1} k_{i j}(\lambda) x_{i}\right] \delta x_{i}=0
$$

Finally, the condition that this equation must hold for any $\delta X_{1}$ yields the algebraic equation system (eqn 11 ).

The finite element stiffness coefficients for the quadrilateral element mapped from a square are calculated as

$$
k_{i j}=\iint_{A}\left\{\Phi_{F}^{i} F^{j}+\Phi_{F_{\theta}}^{i} F_{\theta}^{j}+\Phi_{F_{\phi}}^{i} F_{\phi}^{j}+\Phi_{G}^{i} G^{i}+\Phi_{G}^{i} G_{\theta}^{j}+\Phi_{G_{\phi}}^{i} G_{\phi}^{j}+\Phi_{H}^{i} H^{i}+\Phi_{H_{\phi}}^{i} H_{\theta}^{j}+\Phi_{H_{\phi}}^{i} H_{\phi}\right\} \sin \theta \mathrm{d} \theta \mathrm{d} \phi
$$

in which

$$
\begin{gathered}
\Phi_{F}^{i}=[Q(1-\lambda)+2]\left[(\lambda+2) \rho^{\rho} f^{i}+\left(\rho^{\nu}\right) \theta_{\theta} g^{i}+\rho^{p} g_{\theta}^{i}+\rho^{p} g^{i} \cot \theta+\frac{\rho^{p}}{\sin \theta} h_{\phi}^{i}\right]-2 \lambda(\lambda+2) \rho^{\rho} f^{i}, \quad \Phi_{F_{\phi}}^{i}=\ldots, \ldots \\
\Phi_{H_{\phi}}^{i}=\frac{1}{\sin \theta}\left\{\left[Q(\lambda+2) \rho^{p} f^{i}+\left(\rho^{p}\right) g_{\theta} g^{i}+\rho^{p} g_{\theta}^{i}+\rho^{p} g^{i} \cot \theta+\frac{\rho^{p}}{\sin \theta} h_{\phi}^{i}\right]+2 \frac{\rho^{p}}{\sin \theta} h_{\phi}^{i}+\rho^{p} g^{i} \cot \theta+\rho^{p} f^{i}\right\} .
\end{gathered}
$$

The finite element stiffiness matrix is obtained by mapping the general quadrilateral element in $(\theta, \phi)$ plane into a unit square. The mapping is given by the transformation

$$
\left\{\begin{array}{l}
\theta \\
\phi
\end{array}\right\}=\left[\begin{array}{ll}
\mathbf{B}, & 0 \\
0, & \mathbf{B}
\end{array}\right]\left\{\begin{array}{l}
\mathrm{T} \\
\mathbf{F}
\end{array}\right\}
$$

where

$$
\begin{gathered}
\mathrm{B}=\left(B_{1}, B_{2}, B_{3}, B_{4}\right), \quad B_{i}=\frac{1}{4}\left(1+\theta^{*} \theta^{*}\right)\left(1+\phi^{*} \phi_{i}^{*}\right), \quad(i=k, l, m, n) \\
T=\left(\theta_{k}, \theta_{1}, \theta_{m}, \theta_{n}\right)^{T}, \quad F=\left(\phi_{k}, \phi_{l}, \phi_{m}, \phi_{n}\right)^{T}
\end{gathered}
$$

in which superscript $T$ denotes a transpose: $\left(\theta_{*}^{*}, \phi^{*}\right)$ are the corners $( \pm 1, \pm 1)$ of the unit square, numbered clockwise beginning at $(-1,-1) ;\left(\theta_{i}, \phi_{i}\right)$ are the corresponding corner coordinates of the quadrilateral element; $\left(\theta^{*}, \phi^{*}\right)$ are the coordinates of a general point within the unit square; and $(\theta, \phi)$ are the coordinates of the corresponding point on the quadrilateral element.

Carrying out the foregoing transformation of variables $\theta$ and $\phi$ to $\theta^{*}$ and $\phi^{*}$, we may express the stiffness coefficients given by eqn (12) in the well-known[24] manner:

$$
k_{i j}=\iint_{A} \Psi(\theta, \phi) \mathrm{d} \theta \mathrm{d} \phi=\int_{-1}^{1} \int_{-1}^{1} \Psi *\left(\theta^{*}, \phi^{*}\right) \mathrm{d} \theta^{*} \mathrm{~d} \phi^{*}
$$

in which $\Psi(\theta, \phi)$ is the integrand of eqn (12), and

$$
\Psi^{*}\left(\theta^{*}, \phi^{*}\right)=\Psi\left(\theta^{*}, \phi^{*}\right) J ; \quad J=\left|\begin{array}{ll}
\partial \theta / \partial \theta^{*}, & \partial \phi / \partial \theta^{*} \\
\partial \theta / \partial \phi^{*}, & \partial \phi / \partial \phi^{*}
\end{array}\right|
$$

where $J$ is the Jacobian of the transformation in eqn (B6). The numerical evaluation of the last integral in eqn (B9) is carried out by the Gaussian quadrature formula[24]:

$$
k_{i j}=\sum_{p=1}^{3} \sum_{q=1}^{3} H_{p} H_{q} \Psi^{*}\left(a_{p}, a_{q}\right)
$$

in which $a_{3}=-a_{1}=0.7745 \% 669241483, a_{2}=0, H_{1}=H_{3}=5 / 9, H_{2}=8 / 9$. 


\title{
ADDENDUM TO THE PAPER
}

\section{SURFACE SINGULARITY AND CRACK PROPAGATION†}

\author{
Zdenék P. Bażant and LuIs F. Estenssoro
}

Department of Civil Engineering, Northwestern University Evanston, IL 60201, U.S.A.

The purpose of this Addendum is to provide new experimental results which corroborate the findings of the paper.

As has been theoretically shown in this paper, the front edge of a planar Mode I crack that propagates in an elastic isotropic plate in a plane normal to the plate surfaces must terminate at the surfaces with a certain angle $\beta$ depending only on Poisson ratio $\nu$. As far as comparisons with experimental results are concerned, some limited evidence was shown in Figs. 12 and 13 of the paper. The agreement was essentially qualitative, confirming that for $\nu>0$ angle $\beta$ exceeds $90^{\circ}$ such that the terminal point of crack edge trails in propagation behind the adjacent interior points of crack edge. However, the observed angle in these tests was about $10^{\circ}$ higher than the obtained theoretical value $\beta=102^{\circ}$ for $\nu=0.33$. The discrepancy may have been due to inelastic behavior near the crack edge, particularly due to the fact that these were fatigue tests which were no doubt influenced by cyclic plastic deformation near the edge.

After publication of the paper, more relevant test results were provided to the writers by Professor Stanley T. Rolfe from the University of Kansas at Lawrence. These tests, the photographs of which are exhibited in Figs. 15(a)-(c), with the permission of Professor Rolfe,

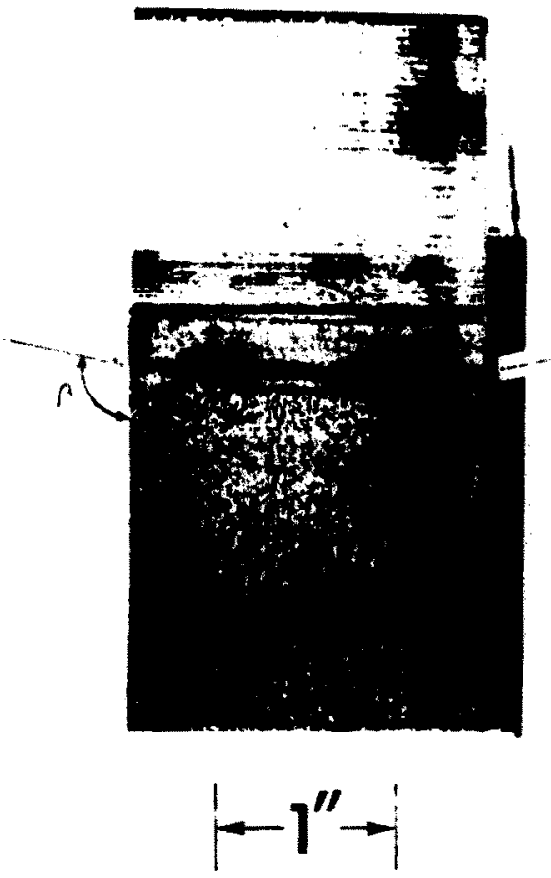

(a)

†Z. P. Bažant and L. F. Estenssoro, Surface singularity and crack propagation. Int. J. Solids Structures 15, 405-426 (1978). 


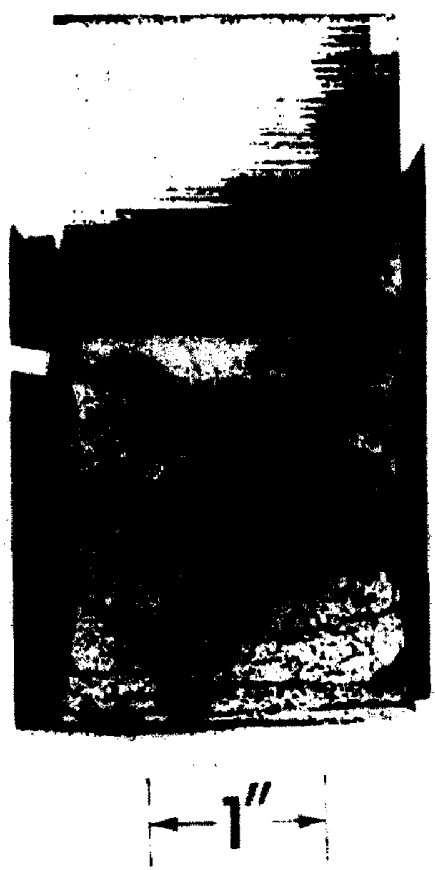

(b)

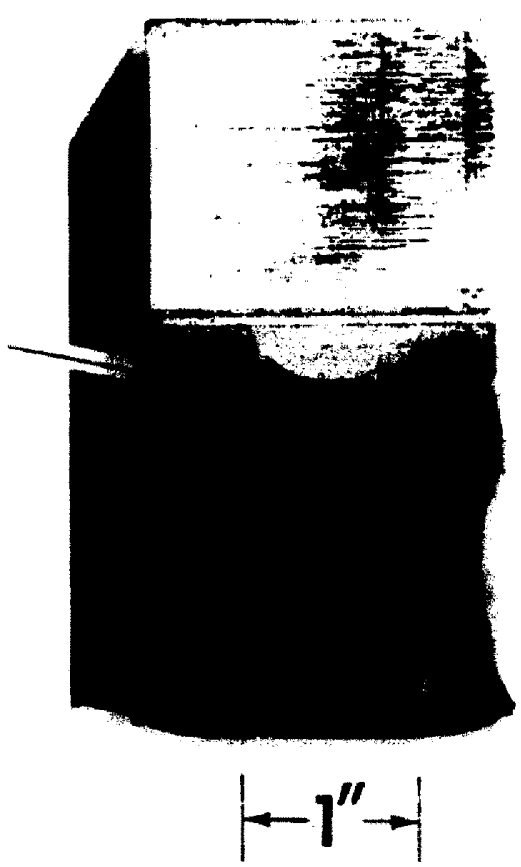

(c)

Fig. $15(a, b, c)$. Three fracture tests of S. T. Rolfe compared with theoretical direction of the crack at the surface points (reproduced with the permission of S. T. Rolfe, University of Kansas at Lawrence). 
were carried out on structural steel in static loading, tested according to the E-399 ASTM Standard. For further comments on this type of test the reader may consult pages 351-353 of Ref.[26].

The theoretical lines of inclination $\beta=101^{\circ}$ with the plate surface have been drawn in Fig. 15. It is seen that they very well agree with the observed terminal directions of the first crack arrest marks, which correspond to an essentially elastic fracture stage. In fact, the theoretical lines do not appear to deviate at all from these observed directions within the resolution of the photographs.

This comparison presents an excellent experimental confirmation of the theory presented in the paper.

\section{REFERENCE}

26. S. T. Rolfe and J. M. Barsom, Fracture and Fatigue Control in Structures. Prentice Hall, Englewood Clifs, New Jersey (1977). 